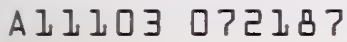

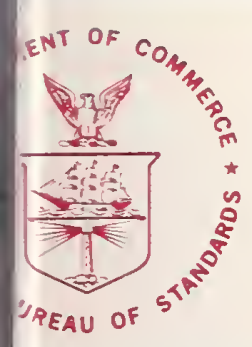

\section{NBS SPECIAL PUBLICATION $\mathbf{5 7 8}$}

U.S. DEPARTMENT OF COMMERCE / National Bureau of Standards

\section{A Catalog of Data Compilations on Photochemical and Photophysical Processes in Solution}




\section{NATIONAL BUREAU OF STANDARDS}

The National Bureau of Standards' ${ }^{1}$ was established by an act of Congress on March 3, 190I. The Bureau's overall goal is to strengthen and advance the Nation's science and technology and facilitate their effective application for public benefit. To this end, the Bureau conducts research and provides: (1) a basis for the Nation's physical measurement system, (2) scientilic and technological services for industry and government, (3) a technical basis for equity in trade, and (4) technical services to promote public safety. The Bureau's technical work is performed by the National Measurement Laboratory, the National Engineering Laboratory, and the Institute for Computer Sciences and Technology.

THE NATIONAL MEASUREMENT LABORATORY provides the national system of physical and chemical and materials measurement; coordinates the system with measurement systems of other nations and furnishes essential services leading to accurate and uniform physical and chemical measurement throughout the Nation's scientitic community, industry, and commerce; conducts materials research leading to improved methods of measurement, standards, and data on the properties of materials needed by industry, commerce, educational institutions, and Government; provides advisory and research services to other Government agencies; develops, produces, and distributes Standard Reference Materials; and provides calibration services. The Laboratory consists of the following centers:

Absolute Physical Quantities ${ }^{2}$ - Radiation Research - Thermodynamics and Molecular Science - Analytical Chemistry - Materials Science.

THE NATIONAL ENGINEERING LABORATORY provides technology and technical services to the public and private sectors to address national needs and to solve national problems; conducts research in engineering and applied science in support of these efforts; builds and maintains competence in the necessary disciplines required to carry out this research and technical service; develops engineering data and measurement capabilities; provides engineering measurement traceability services; develops test methods and proposes engineering standards and code changes; develops and proposes new engineering practices; and develops and improves mechanisms to transter results of its research to the ultimate user. The Laboratory consists of the following centers:

$$
\begin{aligned}
& \text { Applied Mathematics - Electronics and Electrical Engineering }{ }^{2}-\text { Mechanical } \\
& \text { Engineering and Process Technology }{ }^{2}-\text { Building Technology - Fire Research - } \\
& \text { Consumer Product Technology - Field Methods. }
\end{aligned}
$$

THE INSTITUTE FOR COMPUTER SCIENCES AND TECHNOLOGY conducts research and provides scientific and technical services to aid Federal agencies in the selection, acquisition, application, and use of computer technology to improve effectiveness and economy in Government operations in accordance with Public Law 89-306 (40 U.S.C. 759), relevant Executive Orders, and other directives; carries out this mission by managing the Federal Information Processing Standards Program, developing Federal ADP standards guidelines, and managing Federal participation in ADP voluntary standardization activities; provides scientific and technological advisory services and assistance to Federal agencies; and provides the technical foundation for computer-related policies of the Federal Government. The Institute consists of the following centers:

Programming Science and Technology-Computer Systems Engineering.

'Headquarters and Laboratories at Gaithersburg, MD, untess otherwise noted; mailing address Washington, DC 20234.

${ }^{2}$ Some divisions within the center are located at Boułder, CO 80303. 


\section{A Catalog of Data Compilations on Photochemical and Photophysical Processes in Solution}

James G. Brummer, W. Phillip Helman, and Alberta B. Ross

Radiation Chemistry Data Center

Radiation Laboratory

University of Notre Dame

Notre Dame, Indiana 46556

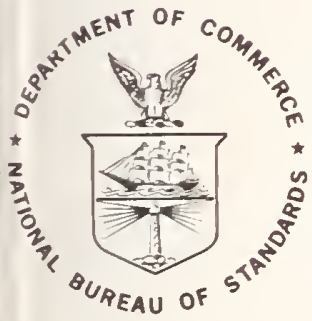

U.S. DEPARTMENT OF COMMERCE, Philip M. Klutznick, Secretary Luther H. Hodges, Jr., Deputy Secretary Jordan J. Baruch, Assistant Secretary for Productivity, Technology and Innovation NATIONAL BUREAU OF STANDARDS, Ernest Ambler, Director 
Library of Congress Catalog Card Number: 80-600117

National Bureau of Standards Special Publication 578 Nat. Bur. Stand. (U.S.), Spec. Publ. 578, 27 pages (Nov. 1980)

CODEN: XNBSAV

\section{U.S. GOVERNMENT PRINTING OFFICE \\ WASHINGTON: 1980}

For sale by the Superintendent of Documents, U.S. Government Printing Office, Washington, D.C. 20402 Price $\$ 1.75$

(Add 25 percent for other than U.S. mailing) 


\section{Foreword}

The National Standard Reference Data System was established in 1963 for the purpose of promoting the critical evaluation and dissemination of numerical data of the physical sciences. The program is coordinated by the Office of Standard Reference Data of the National Bureau of Standards but involves the efforts of many groups in universities, government laboratories, and private industry. The primary aim of the program is to provide compilations of critically evaluated physical and chemical property data. These tables are published in the Journal of Physical and Chemical Reference Data, in the NSRDS.NBS series of the National Bureau of Standards, and through other appropriate channels.

The task of critical evaluation is carried out in various data centers, each with a well-defined technical scope. A necessary preliminary step to the critical evaluation process is the retrieval from the world scientific literature of all papers falling within the scope of the center. Each center, therefore, builds up a comprehensive well-indexed bibliographical file which forms the base for the evaluation task. Bibliographies derived from these files are published when they appear to be of value to research workers and others interested in the particular technical area.

Further information on NSRDS and the publications which form the primary output of the

program may be obtained by writing to the Office of Standard Reference Data, National Bureau of Standards, Washington, DC 20234.

David R. Lide, Jr., Chief

Office of Standard Reference Data 



\title{
A Catalog of Data Compilations on Photochemical and Photophysical Processes in Solution
}

\author{
James G. Brummer, W. Phillip Helman and Alberta B. Ross \\ Radiation Chemistr. Data Center*, Radiation Laboratory. \\ Liniversity of Notre Dame, Notre Dame, Indiana 46556
}

References to compilations and reviews of data on photochemical and photophysical processes in solution have been annotated to indicate subject and data content. Indexes are included for data types, keywords and authors.

Keywords: Data compilations; photochemistry; photophysics; review; solutions.

\section{Introduction}

In 1971 a Report of the CODATA Task Group for Chemical Kinetics ${ }^{1}$ listed 228 reviews, compilations and evaluations of kinetic data which had been published, or which were planned, in preparation or in press. That catalog included on'y a few publications on photochemistry in solution. Since then the Chemical Kinetics Information Center ${ }^{2}$ and other groups sponsored by the National Standard Reference Data System ${ }^{3}$ have carried out a number of compilations and evaluations of gas phase kinetic data relevant to photochemistry. The present survey of availability of data compilations on photochemical and photophysical processes in solution has been undertaken in order to find areas where tabulations and reviews of such data have been made; the catalog will provide a reference aid for locating collections of such data.

"The Radiation Laboratory is operated under Contract EY-76-C02-0038 with the Department of Energy. The Radiation Chemistry Data Center is supported jointly by the National Bureau of Standards, Office of Standard Reference Data and by the Office of Basic Energy Sciences and the Office of Health and Environmental Research of the Department of Energy. This is Radiation Laboratory Document No. NDRL-2062.

${ }^{1} \mathrm{~A}$ Catalog of Compilation and Data Evaluation Activities in Chemical Kinetics, Photochemistry and Radiation Chemistry, by D. Garvin, CODATA Bulletin, Issue No. 3, Dec. 1971, 28p.

${ }^{2}$ National Bureau of Standards, Washington, DC, Dr. Robert Hampson, Jr., Director

${ }^{3}$ See: Critical Evaluation of Data in the Physical Sciences - A Status Report on the National Standard Reference Data System, January 1977. S.A. Rossmassler, Ed., NBS Technical Note 947, May 1977, $84 \mathrm{p}$.
The Radiation Chemistry Data Center collects and indexes current literature within its scope, which has recently been expanded to include photochemical and photophysical processes in solution. The citations herein are part of the RCDC Bibliographic Data Base which contains 45,000 references (mainly since 1966) on radiation chemistry and photochemistry. A careful examination was made of our files and other sources; we hope that readers will call our attention to compilations which should have been included. Although this catalog does not include unpublished works, we should also like to know of compilations in preparation or in press.

The present catalog has been limited to those publications which contain substantial tabulations of data. Some publications here cited contain kinetic data which were obtained by methods other than photochemistry, but which may be relevant to photochemical processes. Compilations are also included on spectroscopy of excited electronic states and transient radicals and radical ions in solution. Reviews have not been cited where data were included mainly for illustration; likewise, textbooks are omitted. References to publications available only in Japanese or Russian have also been omitted. In addition to the references cited here, our files show that several hundred reviews on photochemistry have been published in a wide variety of periodicals, books and serials. There are a number of review series devoted to photochemical topics which are listed in the 
Appendix. Only a few of the reviews in those series are cited here. For current reviews the reader is referred to the references listed in category $\mathrm{Z}$ of the Biweekly List of Papers on Radiation Chemistry and Photochemistry ${ }^{4}$

\section{Arrangement of References and Indexes}

References to the data compilations have been grouped by year of publication. The reference list is followed by author, data type and keyword indexes. Each entry in the reference list has been given a number for convenience in preparing the indexes; the first two digits of the number represent the year of publication. Each entry contains one or more symbols indicating the type of data included. A list of symbols for data type follows this section. Symbols and abbreviations are also given for modifiers which are used in parentheses or as subscripts to the data types to represent processes or states; thus, $\tau_{\mathrm{P}}=$ phosphores. cence lifetime. $\phi_{\mathrm{aq}}=$ quantum yield for aquation, and
$\phi_{\mathrm{F}}=$ fluorescence quantum yield are data identifiers combining a data type with a process.

Following the data identifiers are keywords representing classes of materials, compound types, or types of intermediates. The next line of the entry contains numbers of references, tables and figures. In some cases a further annotation was added. All of the references cover liquid phase solutions; where gas, pure liquid, or aqueous systems were also covered those terms are present as keywords. Indexes have been prepared using the data types and keywords associated with each entry. An author index is also included.

\section{List of Symbols and Abbreviations}

\section{Data Types}

$\begin{array}{ll}\phi & \text { quantum yield } \\ \text { prod. anal. } & \text { product analysis } \\ E & \text { energy level } \\ \Delta E & \text { energy gap } \\ \text { abs. spec. } & \text { absorption spectra } \\ \text { emis. spec. } & \text { emission spectra } \\ \text { excit. spec. } & \text { excitation spectra } \\ \epsilon_{\text {abs }} & \text { absorptivity } \\ \epsilon_{\text {emis }} & \text { emissivity } \\ \lambda_{\text {abs }} & \text { absorption maximum } \\ \lambda_{\text {emis }} & \text { emission maximum } \\ f & \text { oscillator strength } \\ \tau & \text { lifetime } \\ t_{1 / 2} & \text { half-life } \\ k & \text { rate constant } \\ A & \text { preexponential factor } \\ E_{a} & \text { activation energy } \\ K & \text { equilibrium constant } \\ p K & \text { negative logarithm of the } \\ E^{\circ} & \text { acid dissociation constant } \\ I & \text { redox potential } \\ I . P . & \text { luminescence intensity } \\ \mu & \text { ionization potential } \\ R & \text { dipole moment } \\ & \text { energy transfer distance }\end{array}$

\section{Processes and States}

$\mathrm{ab}$

add

aq

B

com

d

$\operatorname{dim}$

dis

disprop

elim

et

ET

EXM

F

$\mathrm{H}-\mathrm{ab}$

hal-ab

ic

isc

isom

$\mathrm{nr}$

ox

abstraction
addition
adduct formulation
association
aquation
biradicals
combination
decay
dimerization
dissociation
disproportionation
elimination
electron transfer
energy transfer
excimer
fluorescence
H abstraction
halogen abstraction
internal conversion
intersystem crossing
isomerization
rearrangement
nonradiative
oxidation

${ }^{4}$ Biweekly List of Papers on Radiation Chemistry and Photochemistry Notre Dame, IN, Radiation Chemistry Data Center, biweekly wit annual indexed cumulation. 
phosphorescence

quartet states

quenching

radiative

racemization red

redox

sub

$\mathrm{S}$

$\mathrm{T}$ reduction

oxidation-reduction

substitution

singlet states

triplet states

\section{References}

64-01 Wilkinson, F. Electronic energy transfer between organic molecules in solution. Advances in Photochemistry, W.A. Noyes, G.S. Hammond and J.N. Pitts, Jr. (eds.), Interscience Publishers, New York, N.Y., 1964, Vol. 3, p.241-268

$k(\mathrm{~T}-\mathrm{T}) ; \tau_{\mathrm{S}} ; \Delta E(\mathrm{~T}-\mathrm{T})$

energy transfer; quenching; aromatics; singlet states; triplet states

98 ref.; 5 tab.; 4 fig.

65-01 Berlman, I.B. Handbook of fluorescence spectra of aromatic molecules. Academic Press, New York, N.Y., 1965, 258p.

$\phi_{\mathrm{F}} ; \tau_{\mathrm{F}} ; \epsilon_{\text {emis }} ; \epsilon_{\mathrm{abs}} ; \lambda_{\text {emis }} ; \lambda_{\text {abs }} ;$ abs. spec.; emis. spec. fluorescence; aromatics; heterocyclic compounds $\sim 100$ ref.; 147 spectra; See later edition [71-04]. Extensive bibliography containing references to 1000 works.

67-01 Adamson, A.W. Photochemistry of complex ions. IV. The role of quartet excited states in the photochemistry of chromium(IlI) complexes. J. Phys. Chem. 71(4): 798-808 (1967)

$\phi_{\text {aq }} ; \phi_{\text {isom }} ; E_{\mathrm{a}}$

quartet states; transition metal complexes; chromium compounds; aqueous

26 ref.; 3 tab.; 3 fig.

67-02 Balzani, V.; Moggi, L.; Scandola, F.; Carassiti, V. Photochemistry of cobalt(III) complexes. Inorg. Chim. Acta Rev. 1: 7-34 (1967)

$\phi_{\text {redox }} ; \phi_{\text {aq }} ; \phi_{\text {isom }} ; \phi_{\text {rac }}$

transition metal complexes; cobalt compounds; aqueous

111 ref.; 6 tab.; 8 fig.
67-03 Birks, J.B.; Munro, I.H. The fluorescence lifetimes of aromatic molecules. Progress in Reaction Kinetics, Porter, G. (ed.), Pergamon Press, New York, NY, 1967, Vol. 4, p.239303

$\phi_{\mathrm{F}} ; k_{\mathrm{r}} ; k_{\mathrm{dis}}(\mathrm{EXM}) ; \tau_{\mathrm{F}}$

solid; polycyclic; dyes; biological materials; aromatics; fluorescence; excimer

257 ref.; 10 tab.; 16 fig.

67-04 Schmillen, A.; Legler, R. Luminescence of organic substances. Landolt-Börnstein, Numerical Data and Functional Relationships in Science and Technology. New Series, Group II: Atomic and Molecular Physics, K.H. Hellwege (ed.), Springer-Verlag, Berlin, 1967, Vol. 3, 416p.

$\phi_{\mathrm{r}} ; k_{q} ; k_{\mathrm{ET}} ; \tau_{\mathrm{r}} ; \lambda_{\text {emis }} ; \lambda_{\text {abs }} ;$ emis. spec.; $\mu ; R$

fluorescence; phosphorescence; transition metal complexes; solid; singlet-triplet transitions; singlet-singlet transitions; quenching; excimer; organic; aromatics; excited states; triplet-triplet transitions; triplet states; energy transfer; singlet states

$\sim 1000$ ref.; many tables and figures; index; main table includes 920 compounds

67-05 Anbar, M.; Neta, P. A compilation of specific bimolecular rate atom; hydroxyl radical bimolecular rate constants for the reactions of hydrated electrons, hydrogen atoms and hydroxyl radicals with inorganic and organic compounds in aqueous solution. Intern. J. Appl. Radiation Isotopes 18: 493523 (1967)

$k$ aqueous; hydrated electron; hydrogen atom; hydroxyl radical

164 ref.; 3 tab. 
68-01 Habersbergerova, A.; Janovsky, 1.; Teply, J. Absorption spectra of intermediates formed during radiolysis and photolysis. Radiat. Res. Rev. 1: 109-81 (1968)

$\epsilon_{\mathrm{abs}} ; \lambda_{\mathrm{abs}} ; t_{1 / 2}$

solvated electrons; radicals; organic; inorganic; solid; heterocyclic compounds; liquid; aliphatics; aromatics

201 ref.; 12 tab.; Extensive compilation of data on radical transients (see [72-01]). Data for both aqueous and nonaqueous solution.

68-02 Valentine, D.,Jr. The photochemistry of cobalt(III) and chromium(III) complexes in solution. Advances in Photochemistry, W.A. Noyes, Jr., G.S. Hammond and J.N. Pitts, Jr. (eds.), John Wiley and Sons, New York, N.Y., 1968, Vol. 6, p.123-92

$\phi_{\mathrm{P}} ; \phi_{\text {aq }} ; \phi_{\mathrm{rac}} ; \phi_{\text {red }} ; \phi_{\text {sub }} ; \tau_{\mathrm{p}} ; E_{\mathrm{a}}$

solid; cobalt compounds; chromium compounds; aqueous; phosphorescence; transition metal complexes

151 ref.; 4 tab.; 7 fig.

68-03 Arnold, D.R. The photocycloaddition of carbonyl compounds to unsaturated systems: The syntheses of oxetanes. Advances in Photochemistry, W.A. Noyes, Jr., G.S. Hammond and J.N. Pitts, Jr. (eds.), John Wiley and Sons, New York, N.Y., 1968, Vol. 6, p.301-423

$\phi_{\text {add }} ; E_{\mathrm{T}} ;$ prod. anal.

alkenes; carbonyl compounds; triplet states; solid;

ketones; aldehydes

166 ref.; 22 tab.; 6 fig.

68-04 Adamson, A.W.; Waltz, W.L.; Zinato, E.; Watts, D.W.; Fleischauer, P.D.; Lindholm, R.D. Photochemistry of transition-metal coordination compounds. Chem. Rev, 68: 541-85 (1968)

$\phi_{\text {rac }} ; \phi_{\text {aq }} ; \phi_{\text {redox }} ; \lambda_{\text {abs }} ; \epsilon_{\text {abs }} ; E_{\text {a }}$

transition metal complexes; cobalt compounds; rhodium compounds; platinum compounds: iron compounds; tungsten compounds; chromium compounds; aqueous

472 ref.; 8 tab.; 13 fig.
69-01 Dilling, W.L. Photochemical cycloaddition reactions of nonaromatic conjugated hydrocarbon dienes and polyenes. Chem. Rev. 69(6): 845-77 (1969)

$\phi_{\text {dim }} ; \phi_{\text {add }} ; E_{\mathrm{T}} ;$ prod. anal.

dienes; polyenes; carbonyl compounds; ketones; triplet states; aromatics

149 ref.; 22 tab.; 3 fig.

69-02 Turro, N.J. Photochemical reactions of organic molecules. Tech. Org. Chem. 14: 133-296 (1969)

$\phi_{\text {ise }} ; \phi_{\text {red }} ; k_{\text {isc }} ; \Delta E(\mathrm{~S}-\mathrm{T}) ; E_{\mathrm{S}} ; E_{\mathrm{T}}$

singlet states; triplet states; carbonyl compounds; alkenes; aromatics; ketones; organic; dienes; solid; polyenes

722 ref.; 11 tab.; 16 fig.

69-03 McGlynn, S.P.; Azumi, T.; Kinoshita, M. Molecular spectroscopy of the triplet state. Prentice-Hall, Inc., Englewood Cliffs, N.J., 1969, 434p.

$\phi_{\mathrm{F}} ; \phi_{\mathrm{P}} ; \phi_{\mathrm{ic}} ; k_{\mathrm{F}} ; k_{\mathrm{iss}} ; \tau_{\mathrm{p}} ; E_{\mathrm{T}} ; \Delta E(\mathrm{~S}-\mathrm{T}) ; \Delta E(\mathrm{~T}-\mathrm{T}) ; f$

triplet states; phosphorescence; aromatics; solid; singlet states; carbonyl compounds; azocompounds; alkenes; benzene; dyes;

heterocyclic compounds; nitro compounds; transition metal complexes; fluorescence

1233 ref.; 434 page book containing numerous tables of data and figures.

69-04 Becker, R.S. Theory and interpretation of fluorescence and phosphorescence. Wiley Interscience, New.York, N.Y. 1969, 283p.

$\emptyset_{\mathrm{F}} ; \emptyset_{\mathrm{p}} ; \emptyset_{\mathrm{lsc}} ; \tau_{\mathrm{F}} ; \tau_{\mathrm{p}} ; \tau_{\mathrm{T}} ; \Delta E(\mathrm{~S}-\mathrm{T}) ; \mathrm{p} K(\mathrm{~S}) ; \mathrm{p} K(\mathrm{~T}) ; \lambda_{\text {emis }}$ aromatics; polycyclic; phosphorescence; fluorescence; heterocyclic compounds; singlet states; triplet states; solid; biological materials; porphyrins; transition metal complexes

525 ref.; 27 tab.; Data for both aqueous and nonaqueous solution. 
70-01 Birks, J.B. Photophysics of Aromatic Molecules. Wiley Interscience, New York. 1970, 704p.

$\phi_{r}: \phi_{\mathrm{isc}} ; \phi_{\mathrm{F}} ; \phi_{\mathrm{T}} ; \phi_{\mathrm{P}} ; k_{\mathrm{q}} ; k_{\mathrm{F}} ; k_{\mathrm{isc}} ; k_{\mathrm{ic}} ; k_{\mathrm{P}} ; k_{\mathrm{dim}}(\mathrm{EXM})$;

$\tau_{\mathrm{T}} ; \tau_{\mathrm{F}} ; \tau_{\mathrm{r}} ; E_{\mathrm{a}} ; E_{\mathrm{T}} ; \Delta E(\mathrm{~T}-\mathrm{T}) ; \Delta E(\mathrm{~S}-\mathrm{T}) ; \epsilon_{\mathrm{abs}}(\mathrm{T}-$

$\mathrm{T}) ; \epsilon_{\mathrm{abs}}(\mathrm{S}-\mathrm{S}): \lambda_{\mathrm{abs}} ; \lambda_{\text {emis }} ;$ I.P.; $R$

aromatics: singlet states; triplet states; excimer;

fluorescence; phosphorescence; exciplex;

energy transfer; quenching; gas; solid: excited states

704 page book citing $\sim 1100$ ref.. Contains numerous tables of data and many figures. Data for both aqueous and nonaqueous solution.

70-02 Dalton, J.C.; Turro, N.J. Photoreactivity of n. $\pi^{*}$ excited states of alkyl ketones. Annu. Rev. Phys. Chem. 21: 499-560 (1970)

$\phi_{\text {isom }} ; \phi_{\text {red }} ; \phi_{\text {elim }} ; \phi\left(\right.$ prod); $k_{\mathrm{q}} ; \tau_{\mathrm{F}} ; \tau_{\mathrm{T}}: \tau_{\mathrm{S}}$; prod. anal.

ketones; aliphatics; fluorescence; singlet states; triplet states; quenching; Norrish type I processes; Norrish type II processes

102 ref.; 14 tab.; 9 fig.

70-03 Birks, J.B. Excimer fluorescence of aromatic compounds. Progress in Reaction Kinetics, G. Porter, K.R. Jennings and P. Suppan (eds.), Pergamon Press, New York, N.Y., 1970, Vol. 5, p.181-272

$\phi_{\mathrm{nr}} ; \phi_{\mathrm{F}} ; \phi_{\mathrm{T}} ; k_{\mathrm{F}} ; k_{\mathrm{dim}}(\mathrm{EXM}) ; k_{\mathrm{dis}}(\mathrm{EXM}) ; k_{\mathrm{nr}} ; k_{\mathrm{ET}} ; k_{\mathrm{q}} ;$ $\tau_{\mathrm{nr}} ; \tau_{\mathrm{F}} ; E_{\mathrm{a}} ; \lambda_{\mathrm{abs}} ; \lambda_{\text {emisis }} ; f$

aromatics; fluorescence: excimer; exciplex; liquid; solid

245 ref.; 23 tab.; 21 fig.

70-04 Vander Donckt, E. Acid-base properties of excited states. Progress in Reaction Kinetics, G. Porter, K.R. Jennings and P. Suppan (eds.), Pergamon Press, New York, N.Y.. 1970, Vol. 5, p.273-99

$\mathrm{p} K(\mathrm{~S}) ; \mathrm{p} K(\mathrm{~T})$

singlet states; triplet states; aromatics; amines; phenols, substituted; carboxylic acids: acidity

98 ref.; 10 tab.; 11 fig.
70-05 Denisov, E.T. Interactions between atoms and radicals in the liquid phase. Russ. Chem. Rev. 39(1): 31-46 (1970)

$k_{\text {com }} ; k_{\text {disprop }} ; k ; E_{\mathrm{a}} ; A$

radicals; alkyl radicals; liquid; alkoxy radicals; phenols, substituted; peroxy radicals; radical processes; aryloxy radicals

165 ref.; 9 tab.; Data for both aqueous and nonaqueous solution.

71-01 De Mayo, P. Enone photoannelation. Acc. Chem. Res. 4(2): 41-7 (1971)

$k_{\mathrm{d}} ; k_{\text {add }} ; k_{\mathrm{q}}$

exciplex; triplet states; ketones; alkenes; alkynes; quenching

61 ref.; 1 tab.

71-02 EngeI, P.S.; Monroe, B.M. Complications in photosensitized reactions. Advances in Photochemistry, J.N. Pitts, Jr., G.S. Hammond, and W.A. Noyes, Jr. (eds.), John Wiley and Sons, New York, N.Y., 1971, Vol. 8, p.245-313

$\phi_{\mathrm{F}} ; \phi_{\mathrm{isc}} ; k_{\mathrm{ET}} ; \tau_{\mathrm{F}} ; \tau_{\mathrm{P}} ; E_{\mathrm{T}} ; E_{\mathrm{S}}$

fluorescence; energy transfer; aromatics; ketones; aldehydes; carbonyl compounds; triplet states; singlet states; solid; phosphorescence

323 ref.; 12 tab.; 7 fig.

71-03 Denisov, E.T. Redox reactions of atoms and radicals with ions in solution. Russ. Chem. Rev. 40(1): 24-33 (1971)

$k_{\mathrm{et}} ; k ; E_{\mathrm{a}} ; A$

hydrogen atom; radicals; metal ions; transition metal complexes; peroxy radicals; alkyl radicals; hydroxyl radical; inorganic; organic

130 ref.; 6 tab.; Data for both aqueous and nonaqueous solution. 
71-04 Berlman, l.B. Handbook of fluorescence spectra of aromatic molecules. Academic Press, New York, N.Y., Second ed., 1971, $473 p$.

$\phi_{\mathrm{F}} ; \tau_{\mathrm{p}} ; \tau_{\mathrm{F}} ; \epsilon_{\mathrm{abs}} ; \epsilon_{\text {emis }} ; \lambda_{\text {emis }} ; \lambda_{\mathrm{abs}} ;$ emis. spec.; abs. spec.; $R$

heterocyclic compounds; polyphenyls; polycyclic; fluorescence; aromatics

255 ref.; 307 spectra; Extensive bibliography containing references to 1000 works. See earlier edition [65-01].

72-01 Habersbergerova, A.; Janovsky, 1.; Kourim, P. Absorption spectra of intermediates formed during radiolysis and photolysis. Part II. Radiat. Res. Rev. 4(2/3): 123-231 (1972)

$\epsilon_{\mathrm{abs}} ; \lambda_{\mathrm{abs}}$

radicals; aromatics; aliphatics; heterocyclic compounds; solvated electrons; solid; liquid; organic; inorganic

410 ref.; 14 tab.; Extensive compilation of data on radical transients (see [68-01] for part I). Data for both aqueous and nonaqueous solution.

72-02 Howard, J.A. Absolute rate constants for reactions of oxyl radicals. Advances in Free-Radical Chemistry, G.H. Williams (ed.), Academic Press, New York, N.Y., 1972, Vol. 4 , p.49-173

$k ; k_{\mathrm{H}-\mathrm{ab}} ; k_{\mathrm{com}} ; k_{\mathrm{ox}} ; k_{\mathrm{ds} \text { sprop }} ; E_{\mathrm{a}} ; K ; A$

radicals; hydroxyl radical; alkoxy radicals; peroxy radicals; triplet states; gas; aromatics; aldehydes; ketones; phenols, substituted; amines; metal ions; nitroxide radicals; carbonyl compounds; aryloxy radicals; aliphatics

300 ref.; 50 tab.; Data for both aqueous and nonaqueous solution.
72-03 Berces, T. The decomposition of aldehydes and ketones. Chemical Kinetics, C.H. Bamford and C.F.H. Tipper (eds.), Elsevier Publishing Co., New York, N.Y., 1972, Vol. 5, p.234-380

$\phi($ prod); prod. anal.

aldehydes; ketones; aliphatics; gas; excited states

333 ref.; 33 tab.; Data for both aqueous and nonaqueous solution.

73-01 Anbar, M.; Bambenek, M.; Ross, A.B. Selected specific rates of reactions of transients from water in aqueous solution. 1. Hydrated electron. NSRDS-NBS-43, May 1973, 59p.

\section{$k$}

aqueous; hydrated electron; organic; inorganic; metal ions; transition metal complexes; aliphatics; aromatics; biological materials 202 ref.; 4 tab Formula index.

73-02 Dorfman, L.M.; Adams, G.E. Reactivity of the hydroxyl radical in aqueous solutions. NSRDS-NBS 46, June, 1973, 59p.

$k_{\mathrm{add}} ; k_{\mathrm{H}-\mathrm{ab}} ; k_{\mathrm{et}}$

hydroxyl radical; aqueous; biological materials; oxide radical ion; radical processes; inorganic; alcohols; carboxylic acids; carbonyl compounds; organic; aliphatics; radicals

320 ref.; 21 tab.

73-03 Kirk, A.D. The photochemistry of chromium(1Il) complexes. Mol. Photochem. 5(2): 127-50 (1973)

$\phi_{\text {aq }} ; E_{\text {a }}$

transition metal complexes; chromium compounds quenching; aromatics; energy transfer

61 ref.; 4 tab.; 6 fig. 
73-04 Scaiano, J.C. Intermolecular photoreductions of ketones. J. Photochem. 2(2): 81-118 (1973)

$\emptyset_{\text {red }} ; k_{\mathrm{q}} ; k_{\mathrm{H}-\mathrm{ab}} ; \tau_{\mathrm{T}} ; \tau_{\mathrm{r}}$

gas; ketones; fluorescence; triplet states; carbonyl compounds; aliphatics; aromatics; amines; quenching; alcohols; boranes

199 ref.; 19 tab.; 3 fig.

73-05 Murov, S.L. Handbook of photochemistry. Dekker, New York, 1973, 272p.

$\phi_{\mathrm{F}} ; \phi_{\mathrm{p}} ; \phi_{\mathrm{isc}} ; k_{\mathrm{q}} ; k_{\mathrm{ET}} ; k_{\mathrm{H}-\mathrm{ab}} ; \tau_{\mathrm{T}} ; \tau_{\mathrm{F}} ; \tau_{\mathrm{p}} ; E_{\mathrm{S}} ; E_{\mathrm{T}} ; \epsilon_{\mathrm{abs}} ; \mu$ quenching; aromatics; ketones; gas; solid: organic; energy transfer; triplet states; singlet states; carbonyl compounds; alkenes

Book of data tables covering materials of interest to photochemists. Bibliography. Indexes. Data for both aqueous and nonaqueous solution.

73-06 Birks, J.B. The spectroscopy of the $\pi$-electronic states of aromatic hydrocarbons. Organic Molecular Photophysics, J.B. Birks (ed.), John Wiley and Sons, New York, N.Y., 1973, Vol. 1, p.1-55

$\phi_{\mathrm{p}} ; k_{\mathrm{isc}} ; k_{\mathrm{F}} ; k_{\mathrm{nr}} ; \tau_{\mathrm{f}} ; \tau_{\mathrm{T}} ; E_{\mathrm{s}} ; E_{\mathrm{T}} ; E_{\mathrm{a}} ; \Delta E(\mathrm{~S}-\mathrm{S}) ; \Delta E(\mathrm{~T}-$ $\mathrm{T}) ; \epsilon_{\mathrm{abs}}(\mathrm{S}-\mathrm{S}) ; \epsilon_{\mathrm{abs}}(\mathrm{T}-\mathrm{T})$

aromatics; singlet states; triplet states; fluorescence; polycyclic; singlet-singlet transitions; singlettriplet transitions; triplet-triplet transitions

98 ref.; 16 tab.

73-07 Labhart, H.; Heinzelmann, W. Triplettriplet absorption spectra of organic molecules. Organic Molecular Photophysics. J.B. Birks (ed.), John Wiley and Sons, New York, N.Y., 1973, Vol. 1, p.297-355

$\Delta E(\mathrm{~T}-\mathrm{T}) ; \epsilon_{\mathrm{abs}}(\mathrm{T}-\mathrm{T})$

triplet-triplet transitions; carbonyl compounds; aromatics; dyes; heterocyclic compounds; porphyrins; biological materials; solid

250 ref.; 9 tab.; 5 fig.
73-08 Engel, P.S.; Steel, C. Photochemistry of aliphatic azo compounds in solution. Acc. Chem. Res. 6: 275-81 (1973)

$\phi(\operatorname{prod})$

azo-compounds; singlet states; aliphatics; triplet states

69 ref.; 3 tab.; 3 fig.

73-09 Chapman, O.L.; Weiss, D.S. Photochemistry of cyclic ketones. Organic Photochemistry, O.L. Chapman (ed.), Marcel Dekker, Inc., New York, N.Y., 1973, Vol. 3, p.197-288

$\phi ; \phi_{\mathrm{p}} ; \phi_{\mathrm{isom}} ; k_{\mathrm{H}-\mathrm{ab}} ; \tau_{\mathrm{T}} ; \tau_{\mathrm{F}} ; \lambda_{\mathrm{abs}} ; \epsilon_{\mathrm{abs}}$

ketones; alkenes; triplet states; singlet states; carbonyl compounds; solid

222 ref.; 14 tab.

73-10 Kirmse, W. Carbene and carbenoid formation and reactions. Chemical Kinetics, C.H. Bamford and C.F.H. Tipper (eds.), Elsevier Scientific Publishing Co., New York, N.Y., 1973, Vol. 9, p.373-4.15

$\phi(\operatorname{prod}) ; k$

carbenes; azo-compounds; alkanes; alkenes; gas; singlet states; liquid; triplet states; biradicals; ethers

140 ref.; 22 tab.

73-11 Berlman, I.B. Energy transfer parameters of aromatic compounds. Academic Press, New York, N.Y., 1973, 379p.

$R$

organics; aromatics; heterocyclic compounds; energy transfer

450 ref.; Extensive tables of donor-acceptor distances for efficient energy transfer.

74-01 Hayon, E.; Simic, M. Acid-base properties of free radicals in solution. Acc. Chem. Res. 7(4): 114-21 (1974)

$\mathrm{p} K$ (radicals)

radicals; acidity; inorganic; organic; alkyl radicals; biological materials; hydroxyalkyl radicals; aqueous

108 ref.; 5 tab.; 1 fig. 
74-02 Wrighton, M. The photochemistry of metal carbonyls. Chem. Rev. 74(4): 401-30 (1974)

$\emptyset_{\mathrm{r}} ; \emptyset_{\mathrm{sub}} ; \varnothing ; \emptyset_{\mathrm{isom}} ; \tau_{\mathrm{r}} ; \lambda_{\mathrm{abs}} ; \lambda_{\text {emis }} ; \epsilon_{\mathrm{abs}} ;$ prod. anal.

metal carbonyls; solid; luminescence; alkenes; dienes; chromium compounds; molybdenum compounds; tungsten compounds; vanadium compounds; niobium compounds; tantalum compounds; iron compounds; ruthenium compounds; osmium compounds; rhenium compounds; transition metal complexes

273 ref.; 25 tab.; 7 fig.

74-03 Denisov, E.T. Liquid-phase reaction rate constants. IFI/Plenum, New York, N.Y., 1974, $771 \mathrm{p}$.

$k_{\mathrm{d} 1 \mathrm{~s}} ; k_{\text {com }} ; k_{\text {isom }} ; k_{\text {sub }} ; k_{\text {hal-ab }} ; k_{\mathrm{H}-\mathrm{ab}} ; k_{\text {add }} ; k_{\text {disprop }} ; E_{\mathrm{a}} ; A$

radicals; radical processes; organic; hydrogen atom; hydroxyl radical; alkyl radicals; aromatics; aliphatics; alkoxy radicals; aryloxy radicals; peroxy radicals; nitroxide radicals; halogen atoms; alkenes

1385 ref.; 114 tab.; The three chapters of this book are entitled (1) Reactions of Molecules, (2) Reactions of Free Atoms and Radicals, and (3) Ionic Homolytic Reactions. Data for both aqueous and nonaqueous solution.

74-04 Hendry, D.G.; Mill, T.; Piszkiewicz, L.; Howard, J.A.; Eigenmann, H.K. A critical review of $\mathrm{H}$-atom transfer in the liquid phase: Chlorine atom, alkyl, trichloromethyl, alkoxy, and alkylperoxy radicals. J. Phys. Chem. Ref. Data 3(4): 937-78 (1974)

$k_{\mathrm{H}-\mathrm{a} b} ; E_{\mathrm{a}} ; A$

carbonyl compounds; radicals; liquid; alkanes; alkenes; silanes; organic; halogen atoms; alcohols; ethers; alkoxy radicals; alkyl radicals; peroxy radicals

149 ref.; 39 tab.; 2 fig.
74-05 Standard fluorescence spectra, 4 Volumes.

Satdler Research Laboratories, Philadelphia, PA, 1974

$\lambda_{\text {emis }}$; emis. spec.; excit. spec.

fluorescence; aromatics

4 volumes containing spectra for 1000 compounds. Data for both aqueous and nonaqueous solution.

75-01 Ross, A.B. Selected specific rates of reactions of transients from water in aqueous solution. Hydrated electron, supplemental data. NSRDS-NBS 43, Supplement, June 1975, 43p.

$k$

aqueous; hydrated electron; organic; inorganic

163 ref.; 3 tab.; Formula index. This volume is a Supplement to [73-01].

75-02 Anbar, M.; Farhataziz Ross, A.B. Selected specific rates of reactions of transients from water in aqueous solution. 11. Hydrogen atom. NSRDS-NBS-51, U.S. Dept. of Commerce, National Bureau of Standards, Washington, D.C., 1975, 56p.

$k$

aqueous; radicals; hydrogen atom; inorganic; organic

219 ref.; 4 tab.; Formula index.

75-03 Khudyakov, I.V.; Kuz'min, V.A. Short-lived phenoxy- and semiquinone radicals. Russ. Chem. Rev. (Engl. Transl.) 44(10): 801-15 (1975)

$k_{\text {com }} ; k_{\text {disprop }} ; \mathrm{p} K($ radicals $) ; \epsilon_{\mathrm{abs}} ; \lambda_{\mathrm{abs}}$ aryloxy radicals; semiquinones; phenols, substituted; quinones; metal ions

205 ref.; 4 tab.; Data for both aqueous and nonaqueous solution. 
75-04 Ermolaev, V.L.; Sveshnikova, E.;

Shakhverdov, T.A. Energy transfer between organic molecules and transition metal ions.

Russ. Chem. Rev. 44(1): 26-40 (1975)

$\emptyset_{\mathrm{aq}} ; k_{\mathrm{q}} ; k_{\mathrm{ET}} ; E_{\mathrm{T}} ; R$

fluorescence; phosphorescence; energy transfer; metal ions; quenching; triplet states; singlet oxygen; transition metal complexes; polycyclic; solid; flavins; aromatics

110 ref.; 11 tab.; 2 fig.; Data for both aqueous and nonaqueous solution.

75-05 Cundall, R.B.; Ogilvie, S.McD. The photophysics of benzene in fluid media. Organic Molecular Photophysics. Volume 2., J.B. Birks (ed.), John Wiley \& Sons. New York, 1975, p.33-93

$\phi_{\mathrm{F}} ; \phi_{\mathrm{F}}(\mathrm{EXM}) ; \quad \phi_{\mathrm{T}} ; \quad \phi_{\mathrm{ic}} ; k_{\mathrm{F}} ; k_{\mathrm{ic}} ; k_{\mathrm{isc}} ; \tau_{\mathrm{F}} ; \tau_{\mathrm{r}} ; \tau_{\mathrm{nr}} ;$ $\tau_{\mathrm{F}}(\mathrm{EXM}) ; \lambda_{\text {abs }}(\mathrm{S}-\mathrm{S}) ; \epsilon_{\mathrm{abs}}(\mathrm{S}-\mathrm{S})$

benzene; gas; deuterium compounds; fluorescence; singlet states; triplet states; liquid; excimer

196 ref.; 11 tab.; 15 fig.; Data for both aqueous and nonaqueous solution.

75-06 Wilkinson, F. Triplet quantum yields and singlet-triplet intersystem crossing. Organic Molecular Photophysics. Volume 2., J.B. Birks (ed.), John Wiley \& Sons, New York, 1975, p.95-158

$\phi_{p} ; \phi_{\text {isc }} ; \phi_{\mathrm{F}} ; \phi_{\mathrm{T}} ; \phi_{\mathrm{F}}(\mathrm{EXM}) ; k_{\text {isc }} ; \tau_{\mathrm{F}} ; R$

energy transfer; triplet states; aromatics; triplettriplet transitions; quenching; solid; excimer; singlet states; carbonyl compounds; heterocyclic compounds; porphyrins; dyes; amines

252 ref.; 12 tab.; 15 fig.
75-07 Birks, J.B. Photophysics of aromatic molecules - a postscript. Organic Molecular Photophysics. Volume 2., J.B. Birks (ed.), John Wiley \& Sons, New York, 1975, p.409613

$\phi_{\mathrm{F}} ; \phi_{\mathrm{P}} ; \phi_{\mathrm{T}} ; k_{\mathrm{r}} ; k_{\mathrm{nr}} ; k_{\mathrm{isc}} ; k_{\mathrm{q}} ; \tau_{\mathrm{F}} ; \tau_{\mathrm{P}} ; \tau_{\mathrm{T}} ; E_{\mathrm{T}} ; E_{\mathrm{S}} ; E_{\mathrm{a}} ;$ $\lambda_{\text {abs }} ; \lambda_{\text {emis }} ; \epsilon_{\text {emis }} ; \mu$

aromatics; polycyclic; gas; liquid; excimer; singlet states; triplet states; fluorescence; phosphorescence; quenching; solid; alkanes; exciplex

618 ref.; 58 tab.; 20 fig.; Sequel, covering work published subsequent to [70-01].

75-08 Balzani, V.; Moggi, L.; Manfrin, M.F.; Bolletta, F.; Laurence, G.S. Quenching and sensitization processes of coordination compounds. Coord. Chem. Rev. 15(4): 321433 (1975)

$k_{\mathrm{ET}} ; k_{\mathrm{q}} ; E_{\mathrm{T}}$

iron compounds; molybdenum compounds; ruthenium compounds; rhodium compounds; platinum compounds; cobalt compounds; copper compounds; transition metal complexes; quenching; energy transfer; aromatics; chromium compounds;

luminescence; zinc compounds; solid; manganese compounds; nickel compounds; rare-earth metal compounds; silver compounds; vanadium compounds; mercury compounds; iridium compounds; palladium compounds; excited states

216 ref.; 7 tab.; 8 fig.; Extensive catalog of excited states of coordination compounds including their modes of relaxation to the ground state. Data for both aqueous and nonaqueous solution.

76-01 Ermolaev, V.L.; Sveshnikova, E.;

Shakhverdov, T.A. Investigation of the formation of complexes of organic molecules and lanthanide ions in solutions by the electronic energy transfer method. Russ. Chem. Rev. 45(10): 896-912 (1976)

$k_{\mathrm{q}} ; k_{\mathrm{ET}} ; \tau_{\mathrm{F}} ; R$

energy transfer; luminescence; rare-earth metal ions; quenching; aromatics; fluorescence; dyes; ketones; triplet states

98 ref.; 13 tab.; 9 fig.; Data for both aqueous and nonaqueous solution. 
76-02 Shlyapintokh, V.Ya.; Ivanov, V.B. The quenching of singlet oxygen. Russ. Chem. Rev. 45(2): 99-110 (1976)

$k_{\mathrm{d}} ; k_{\mathrm{q}} ; k_{\mathrm{ox}}$

quenching; singlet oxygen; alkenes; aromatics; amines; transition metal complexes; gas; energy transfer

77 ref.; 11 tab.; 3 fig.; Data for both aqueous and nonaqueous solution.

76-03 lreland, J.F; Wyatt, P.A.H. Acid-base properties of electronically excited states of organic molecules. Advances in Physical Organic Chemistry, V. Gold and D. Bethell (eds.), Academic Press, New York, N.Y., 1976, Vol. 12, p.131-221

$k_{\text {dis }} ; k_{\text {add }} ; \mathrm{p} K(\mathrm{~S}) ; \mathrm{p} K(\mathrm{~T})$

singlet states; triplet states; acidity; aromatics; phenols, substituted; organic; aqueous; heterocyclic compounds; carbonyl compounds; proton transfer

$\sim 260$ ref.; 17 tab.; 13 fig.

76-04 Bellus, D. Quenchers of singlet oxygen. A critical review. Singlet Oxygen, Reactions with Organic Compounds and Polymers, B. Ranby and J.F. Rabek (eds.), John Wiley \& Sons, New York, N.Y., 1976, p.61-110

$k_{\mathrm{q}} ; k_{\mathrm{ox}} ; k_{\mathrm{d}}$

singlet oxygen; biological materials; energy transfer; amines; quenching; polyenes; phenols, substituted; organic; inorganic; transition metal complexes

231 ref.; 10 tab.; 5 fig.; Data for both aqueous and nonaqueous solution.

76-05 Gollnick, K. Mechanism and kinetics of chemical reactions of singlet oxygen with organic compounds. Singlet Oxygen,

Reactions with Organic Compounds and Polymers, B. Ranby and J.F. Rabek (eds.), John Wiley \& Sons, New York, N.Y., 1976, p.111-34

$k_{\mathrm{q}} ; k_{\mathrm{ox}} ; \tau_{\mathrm{d}}$

singlet oxygen; gas; organic; amines; dienes; aromatics; heterocyclic compounds; biological materials; alkenes

150 ref.; 10 tab.
77-01 Farhataziz; Ross, A.B. Selected specific rates of reactions of transients from water in aqueous solution. III. Hydroxyl radical and perhydroxyl radical and their radical ions. NSRDS-NBS-59, Jan. 1977, 122p.

$k$

aqueous; hydroxyl radical; perhydroxyl radical; radicals; oxide radical ion; superoxide ion; inorganic; organic

$\sim 460$ ref.; 6 tab.; Formula index.

77-02 Martynov, I.Yu.; Demyashkevich, A.B.; Uzhinov, B.M.; Kuz'min, M.G. Proton transfer reactions in the excited electronic states of aromatic molecules. Russ. Chem. Rev. 46(1): 1-15 (1977)

$\phi ; k_{\text {add }} ; k_{\mathrm{q}} ; \mathrm{p} K(\mathrm{~S}) ; \mathrm{p} K(\mathrm{~T})$

singlet states; triplet states; aromatics; phenols, substituted; amines; proton transfer; heterocyclic compounds; acidity; carboxylic acids

186 ref.; 6 tab.; 3 fig.; Data for both aqueons and nonaqueous solution.

77-03 von Sonntag, C.; Schuchmann, H.P. Photolysis of saturated alcohols, ethers, and amines. Advances in Photochemistry, J.N. Pitts, Jr., G.S. Hammond and K. Gollnick (eds.), Wiley, New York, 1977, Vol. 10, p.59145.

$\phi\left(\right.$ prod); $\epsilon_{\text {abs }} ;$ prod. anal.

alcohols; ethers; amines; liquid; aqueous; gas; solid; aliphatics

295 ref.; 27 tab.; Data for both aqueous and nonaqueous solution.

77-04 Cundall, R.B.; Robinson, D.A.; Pereira, L.C. Excitation and deexcitation of benzene. Advances in Photochemistry, J.N. Pitts, Jr., G.S. Hammond and K. Gollnick (eds.), Wiley, New York, 1977, Vol. 10, p.147-219

$\phi_{\mathrm{F}} ; \phi_{\mathrm{T}} ; \phi_{\mathrm{F}}(\mathrm{EXM}) ; k_{\mathrm{F}} ; \tau_{\mathrm{F}} ; \tau_{\mathrm{P}} ; \tau(\mathrm{EXM}) ;$ abs. spec.; emis. spec.; $I$

benzene; singlet states; triplet states; gas; fluorescence; excimer; solid

288 ref.; 15 tah.; 10 fig. 
77-05 Bock, C.R.; Koerner von Gustorf, E.A. Primary photoprocesses of organo-transition metal compounds. Advances in Photochemistry, J.N. Pitts, Jr., G.S. Hammond and K. Gollnick (eds.), Wiley, New York, 1977, Vol. 10, p.221-310

$\phi_{\text {sub }} ; \phi_{\text {isom }} ; \phi_{\text {add }} ; \phi(\operatorname{prod}) ; \phi_{\mathrm{r}} ; k_{\text {add }} ; k_{\text {dis }} ; k_{\mathrm{q}} ; k_{\mathrm{r}} ; k_{\mathrm{nr}} ; \tau_{\mathrm{r}} ;$ $\lambda_{\text {emis }}$

triplet states; transition metal complexes; solid; metal carbonyls; luminescence; quenching; metal-metal bonded complexes

262 ref.; 13 tab.; 12 fig.

77-06 De Schryver, F.C.; Boens, N.; Put, J. Excited state behavior of some bichromophoric systems. Advances in Photochemistry, J.N. Pitts, Jr., G.S. Hammond and K. Gollnick (eds.), Wiley, New York, 1977, Vol. 10, p.359-465

$\phi_{\mathrm{F}} ; \phi_{\text {isom }} ; \phi_{\mathrm{isc}} ; k_{\mathrm{isom}} ; k_{\mathrm{ET}} ; \lambda_{\mathrm{emis}}$

singlet states; triplet states; excimer; alkenes;

fluorescence; energy transfer; ketones; polymerization; aromatics

224 ref.; 24 tab ; 13 fig.

78-01 Buxton, G.V.; Sellers, R.M. Compilation of rate constants for the reactions of metal ions in unusual valency states. NSRDS-NBS 62, 1978, 78p.

\section{$k$}

aqueous; transition metal complexes; metal ions; radicals

96 ref.; 25 tab.

78-02 Maciejewski, A.; Matuszewski, B. Intersystem crossing quantum yield $\phi_{\text {isc }}$ of electronically excited molecules. Part 11. Effect of some physicochemical factors on the values of $\phi_{\text {isc }}$ and comparison of organic compounds for which these values were determined. Wiad. Chem. 32(3): 151-65 (1978)

$\phi_{\text {Isc }}$

singlet states; triplet states

146 ref.; 1 table listing 200 compounds with references in which data may be found.
78-03 Swallow, A.J. Reactions of free radicals produced from organic compounds in aqueous solution by means of radiation. Prog. React. Kinet. 9(3/4): 195-366 (1978)

$k ; k_{\text {com }} ; k_{\text {add }} ; k_{\text {el }} ; k_{\text {elim }} ; \mathrm{p} K$ (radicals); $E_{\mathrm{a}} ; E^{\circ}$

aqueous; radicals; radical processes; organic; alkyl radicals; metal ions; proton transfer; hydroxyalkyl radicals; inorganic; semiquinones; biological materials

360 ref.; 58 tab.

78-04 Scaiano, J.C.; Lissi, E.A.; Encina, M.V. Chemistry of the biradicals produced in the Norrish type II reaction. Rev. Chem. lntermediates 2: 139-95 (1978)

$\phi_{\text {dis }} ; \phi_{\text {isom }} ; k_{\mathrm{H}-\mathrm{ab}} ; k_{\mathrm{et}} ; k ; k_{\text {add }} ; \tau_{\mathrm{T}} ; \tau(\mathrm{B}) ; E_{\mathrm{a}} ; A$

biradicals; Norrish type 11 processes; ketones; triplet states; singlet states; gas; aliphatics; aromatics

288 ref.; 20 tab.; 3 fig.

78-05 Balzani, V.; Bolletta, F.; Gandolfi, M.T. Maestri, M. Bimolecular electron transfer reactions of the excited states of transition metal complexes. Top. Curr. Chem. 75: 1-64 (1978)

$k_{\mathrm{q}} ; \tau_{\mathrm{r}} ; E^{\circ} ; \Delta E ; \lambda_{\text {abs }}$

quenching; chromium compounds; ruthenium compounds; transition metal complexes; osmium compounds; iridium compounds; aromatics; uranium compounds

314 ref.; 6 tab.; 22 fig.

78-06 Bensasson, R.; Land, E.J. Physical properties of excited states: A general method for measuring triplet-triplet extinction coefficients, singlet-triplet intersystem crossing efficiencies, and related parameters. Photochemical and Photobiological Reviews, K.C. Smith(ed.), Plenum Press, New York, N.Y., 1978, Vol 3, p.163-91

$\emptyset_{\text {isc }} ; \lambda_{\text {abs }}(\mathrm{S}-\mathrm{S}) ; \lambda_{\mathrm{abs}}(\mathrm{T}-\mathrm{T}) ; \epsilon_{\mathrm{abs}}(\mathrm{S}-\mathrm{S}) ; \epsilon_{\mathrm{abs}}(\mathrm{T}-\mathrm{T})$

triplet states; singlet states; aromatics; polycyclic; biological materials

68 ref.; 4 tab.; Data for both aqueous and nonaqueous solution. 
79-01 Ross, A.B.; Neta, P. Rate constants for reactions of inorganic radicals in aqueous solution. NSRDS-NBS 65, 1979, 55p.

$k$

aqueous; radicals; inorganic; organic; aliphatics; aromatics

95 ref.; 9 tab.; Data for over 560 radical reactions. Formula index.

79-02 Fox, M.A. The photoexcited states of organic anions. Chem. Rev. 79(3): 253-73 (1979)

$\lambda_{\text {abs }}$ carbanions; radicals; alkali metal ions

279 ref.; 7 tab.; 3 fig.; Data for both aqueous and nonaqueous solution.
79-03 Bellus, D. Physical quenchers of singlet molecular oxygen. Advances in Photochemistry, J.N. Pitts, Jr., G.S. Hammond and K. Gollnick (eds.), John Wiley and Sons, New York, N.Y., 1979, Vol. 11, p.105-205

$k ; k_{\mathrm{q}} ; k_{\mathrm{d}} ; \tau_{\mathrm{d}} ; E_{\mathrm{T}}$

singlet oxygen; organic; quenching; alkenes; amines; biological materials; phenols, substituted; transition metal complexes; heterocyclic compounds

332 ref.; 11 tab.; 7 fig.; Data for both aqueous and nonaqueous solution.

79-04 Gollnick, K.; Kuhn, H.J. Ene-reactions with singlet oxygen. Singlet Oxygen, H.H. Wasserman and R.W. Murray (eds.), Academic Press, New York, N.Y., 1979, Vol. 40 of Organic Chemistry, p. 287-427

$k ; \tau_{\mathrm{d}} ; E_{\mathrm{a}} ;$ prod. anal.; I.P.

alkenes; singlet oxygen; gas

286 ref.; 15 tab.; 4 fig.; Includes an extensive catalog of product yields for the reaction of singlet oxygen with various alkenes.

\section{Data Index}

$\phi$

73-09 74-02 77-02

$\phi($ prod $)$

70-02 72-03 73-08 73-10 77-03 77-05

$\phi_{\mathrm{F}}$

65-01 67-03 69-03 69-04. 70-01 70-03

71-02 71-04 73-05 75-05 75-06 75-07

77-04. 77-06

$\phi_{\mathrm{F}}(\mathrm{EXM})$

75-05 75-06 77-04

$\phi_{\mathrm{P}}$

68-02 69-03 69-04 70-01 73-05 73-06

73-09 75-06 75-07

$\phi$

67-04 70-01 74-02 77-05 $\phi_{\mathrm{nr}}$

70-03

$\phi_{\text {isc }}$

69-02 69-04 70-01 71-02 73-05 75-06

77-06 78-02 78-06

$\phi_{\text {ic }}$

69-03 75-05

$\phi_{\mathrm{T}}$

70-01 70-03 75-05 75-06 75-07 77-04.

$\phi_{\text {add }}$

68-03 69-01 77-05

$\phi_{\text {aq }}$

67-01 67-02 68-02 68-04 73-03 75-04 
$\phi_{\text {dim }}$

69-01

$\phi_{\text {dis }}$

78-04

$\phi_{\text {elim }}$

70-02

$\phi_{\text {isom }}$

67-01 67-02 70-02 73-09 74-02 77-05

$77-0678-04$

$\phi_{\mathrm{rac}}$

67-02 68-02 68-04

$\phi_{\text {red }}$

68-02 69-02 70-02 73-04

$\phi_{\text {redox }}$

67-02 68-04

$\phi_{\text {sub }}$

68-02 74-02 77-05

prod. anal.

68-03 69--01 70-02 72-03 74-02 77-03

79-04

$E_{\mathrm{s}}$

69-02 71-02 73-05 73-06 75-07

$E_{\mathrm{T}}$

68-03 69-01 69-02 69-03 70-01 71-02 73-05 73-06 75-04 75-07 75-08 79-03

$\Delta E$

78-05

$\Delta E(\mathrm{~S}-\mathrm{S})$

73-06

$\Delta E(\mathrm{~S}-\mathrm{T})$

69-02 69-03 69-04 70-01

$\Delta E(\mathrm{~T}-\mathrm{T})$

64-01 69-03 70-01 73-06 73-07

abs. spec.

65-01 71-04 77-04 emis. spec.

65-01 67-04 71-04 74-05 77-04

excit. spec.

74-05

$\epsilon_{\mathrm{abs}}$

65-01 68-01 68-04 71-04 72-01 73-05 73-09 74-02 75-03 77-03

$\epsilon_{\mathrm{abs}}(\mathrm{S}-\mathrm{S})$

70-01 73-06 78-06

$\epsilon_{\mathrm{abs}}(\mathrm{T}-\mathrm{T})$

70-01 73-06 73-07 78-06

$\epsilon_{\text {emis }}$

65-01 71-04 75-07

$\lambda_{\text {abs }}$

65-01 67-04 68-01 68-04 70-01 70-03 71-04 72-01 73-09 74-02 75-03 75-07 78-05 79-02

$\lambda_{\text {abs }}(S-S)$

75-05 78-06

$\lambda_{\text {abs }}(\mathrm{T}-\mathrm{T})$

78-06

$\lambda_{\text {emis }}$

65-01 67-04 69-04 70-01 70-03 71-04

74-02 74-05 75-07 77-05 77-06

69-03 70-03

$\tau_{\mathrm{F}}$

65-01 67-03 69-04 70-01 70-02 70-03 71-02 71-04 73-05 73-09 75-05 75-06 75-07 76-01 77-04

$\tau_{\mathrm{F}}(\mathrm{EXM})$

75-05

$\tau_{\mathrm{P}}$

68-02 69-03 69-04. 71-02 71-04. 73-05 75-07 77-04

$\tau_{\text {r }}$

67-04 70-01 73-04 73-06 74-02 75-05 77-05 78-05 


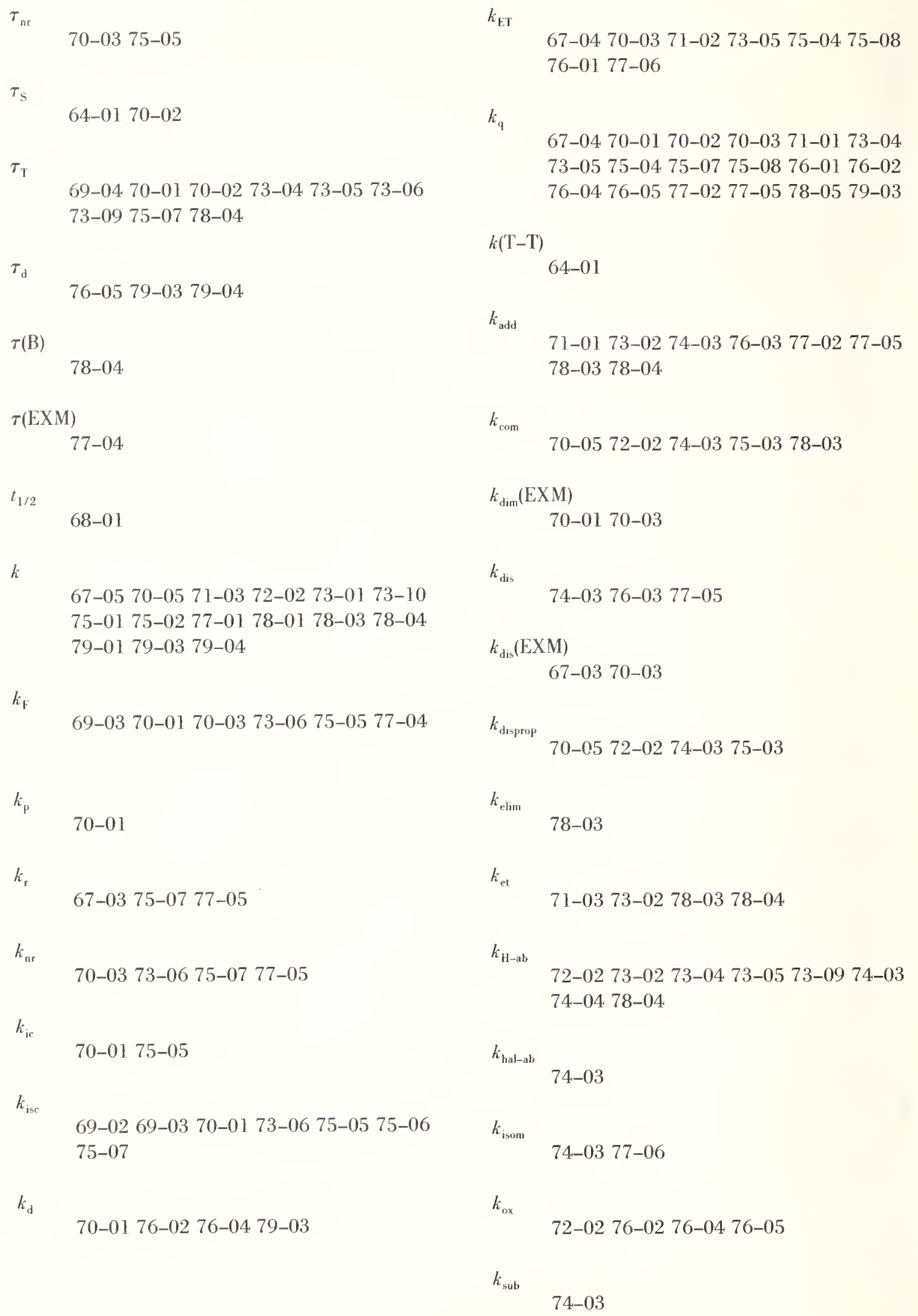


A

70-05 71-03 72-02 74-03 74-04. 78-04.

$E_{\mathrm{a}}$

67-01 68-02 68-04 70-01 70-03 70-05

71-03 72-02 73-03 73-06 74-03 74-04.

75-07 78-03 78-04 79-04

K

72-02

$\mathrm{p} K(\mathrm{~S})$

69-04 70-04 76-03 77-02

$\mathrm{p} K(\mathrm{~T})$

69-04 70-04 76-03 77-02 $\mathrm{p} K$ (radicals)

74-01 75-03 78-03

$E^{\circ}$

78-03 78-05

I

77-04

I.P.

70-01 79-04

$\mu$

67-04 73-05 75-07

$R$

67-04 70-01 73-01 75-04. 75-06 76-01

\section{Keyword Index}

acidity

70-04 74-01 76-03 77-02

alcohols

73-02 73-04 74-04 77-03

aldehydes

$$
\text { 68-03 71-02 72-02 72-03 }
$$

aliphatics

68-01 70-02 72-01 72-02 72-03 73-01

73-02 73-04 73-08 74-03 77-03 78-04

79-01

alkali metal ions

79-02

alkanes

73-10 74-04 75-07

alkenes

68-03 69-02 69-03 71-01 73-05 73-09

73-10 74-02 74-03 74-04 76-02 76-05

77-06 79-03 79-04

(see dienes, polyenes)

alkoxy radicals

70-05 72-02 74-03 74-04. alkyl radicals

70-05 71-03 74-01 74-03 74-04. 78-03

alkynes

$$
\text { 71-01 }
$$

amines

70-04 72-02 73-04 75-06 76-02 76-04. 76-05 77-02 77-03 79-03

aqueous

67-01 67-02 67-05 68-02 68-04 73-01

73-02 74-01 75-01 75-02 76-03 77-01 77-03 78-01 78-03 79-01

aromatics

64-01 65-01 67-03 67-04. 68-01 69-01

69-02 69-03 69-04 70-01 70-03 70-04

71-02 71-04 72-01 72-02 73-01 73-03

73-04 73-05 73-06 73-07 73-11 74-03

74-05 75-04 75-06 75-07 75-08 76-01

76-02 76-03 76-05 77-02 77-06 78-04.

78-05 78-06 79-01

(see benzene, dyes, heterocyclic compounds, phenols, substituted, polycyclic, polyphenyls) 
aryloxy radicals

70-05 72-02 74-03 75-03

azo-compounds

69-03 73-08 73-10

benzene

$$
\text { 69-03 75-05 77-04 }
$$

biological materials

$$
\begin{aligned}
& \text { 67-03 69-04 73-01 73-02 73-07 74-01 } \\
& 76-04.76-05 \text { 78-03 78-06 79-03 }
\end{aligned}
$$

biradicals

$$
\text { 73-10 78-04 }
$$

boranes

$$
\text { 73-04 }
$$

carbanions

$$
\text { 79-02 }
$$

carbenes

$$
73-10
$$

carbonyl compounds

$$
\begin{aligned}
& 68-03 \text { 69-01 69-02 69-03 71-02 72-02 } \\
& 73-02 \text { 73-04 73-05 73-07 73-09 74-04 } \\
& 75-0676-03
\end{aligned}
$$

(see aldehydes, carboxylic acids, ketones, metal carbonyls, quinones)

carboxylic acids

$$
\text { 70-04 73-02 77-02 }
$$

chromium compounds

$$
\begin{aligned}
& \text { 67-01 68-02 68-04, 73-03 74-02 75-08 } \\
& 78-05
\end{aligned}
$$

cobalt compounds

$$
\text { 67-02 68-02 68-04 75-08 }
$$

copper compounds

$$
\text { 75-08 }
$$

deuterium compounds

$$
\text { 75-05 }
$$

dienes

$$
\text { 69-01 69-02 74-02 76-05 }
$$

dyes

$$
\text { 67-03 69-03 73-07 75-06 76-01 }
$$

energy transfer

64-01 67-04 70-01 71-02 73-03 73-05

73-11 75-04 75-06 75-08 76-01 76-02

76-04 77-06

ethers

$$
\text { 73-10 74-04. 77-03 }
$$

excimer

67-03 67-04. 70-01 70-03 75-05 75-06

75-07 77-04. 77-06

exciplex

$$
\text { 70-01 70-03 71-01 75-07 }
$$

excited states

$$
\text { 67-04 70-01 72-03 75-08 }
$$

(see excimer, exciplex, quartet states, singlet oxygen, singlet states, triplet states)

flavins

$$
\text { 75-04 }
$$

fluorescence

$$
\begin{aligned}
& 65-0167-03 \text { 67-04. 69-03 69-04. 70-01 } \\
& 70-02 \text { 70-03 71-02 71-04. 73-04. 73-06 } \\
& 74-05 \text { 75-04. 75-05 75-07 76-01 77-04 } \\
& 77-06
\end{aligned}
$$

gas

70-01 72-02 72-03 73-04 73-05 73-10 75-05 75-07 76-02 76-05 77-03 77-04 78-04 79-04

halogen atoms

74-03 74-04

heterocyclic compounds

65-01 68-01 69-03 69-04. 71-04. 72-01

73-07 73-11 75-06 76-03 76-05 77-02 79-03

(see dyes, flavins, porphyrins)

hydrated electron

67-05 73-01 75-01

hydrogen atom

$$
\text { 67-05 71-03 74-03 75-02 }
$$

hydroxyalkyl radicals

74-01 78-03 
hvdroxyl radical

$$
\text { 67-05 71-03 72-02 73-02 74-03 77-01 }
$$

inorganic

68-01 71-03 72-01 73-01 73-02 74-01

75-01 75-02 76-04 77-01 78-03 79-01

(see metal ions, metal-metal bonded

complexes, rare-earth metal comounds,

rare-earth metal ions, silanes, transition

metal complexes. specific metal complexes)

iridium compounds

75-08 78-05

iron compounds

$$
\text { 68-04 74-02 75-08 }
$$

ketones

68-03 69-01 69-02 70-02 71-01 71-02

72-02 72-03 73-04 73-05 73-09 76-01

$77-0678-04$

liquid

68-01 70-03 70-05 72-01 73-10 74-04.

75-05 75-07 77-03

luminescence

74-02 75-08 76-01 77-05

(see fluorescence, phosphorescence)

manganese compounds

$75-08$

mercury compounds

$$
\text { 75-08 }
$$

metal carbonyls

$$
\text { 74-02 77-05 }
$$

metal ions

71-03 72-02 73-01 75-03 75-04 78-01

78-03

metal-metal bonded complexes

$$
\text { 77-05 }
$$

molybdenum compounds

74-02 75-08

nickel compounds

$75-08$ niobium compounds

74-02

nitro compounds

$$
\text { 69-03 }
$$

nitroxide radicals

72-02 74-03

Norrish type 1 processes

$70-02$

Norrish type 11 processes

70-02 78-04

organic

67-04 68-01 69-02 71-03 72-01 73-01

73-02 73-05 73-11 74-01 74-03 74-04

75-01 75-02 76-03 76-04 76-05 77-01

78-03 79-01 79-03

(see aliphatics, aromatics, specific

compound class i.e. alcohols, ketones,

heterocyclic compounds, quinones,

etc.....)

osmium compounds

74-02 78-05

oxide radical ion

$$
\text { 73-02 77-01 }
$$

palladium compounds

$$
\text { 75-08 }
$$

perhydroxyl radical

77-01

peroxy radicals

70-05 71-03 72-02 74-03 74-04

phenols, substituted

70-04 70-05 72-02 75-03 76-03 76-04

77-02 79-03

phosphorescence

67-04 68-02 69-03 69-04: 70-01 71-02

75-04. 75-07

platinum compounds

68-04. 75-08 
polycyclic

67-03 69-04 71-04 73-06 75-04 75-07

78-06

polyenes

$$
\text { 69-01 69-02 76-04 }
$$

polymerization

$77-06$

polyphenyls

$$
\text { 71-04 }
$$

porphyrins

69-04 73-07 75-06

proton transfer

76-03 77-02 78-03

quartet states

67-01

quenching

$$
\begin{aligned}
& \text { 64-01 67-04 70-01 70-02 71-01 73-03 } \\
& 73-0473-05 \text { 75-04 75-06 75-07 75-08 } \\
& 76-01 \text { 76-02 76-04 77-05 78-05 79-03 } \\
& \text { (see energy transfer) }
\end{aligned}
$$

quinones

$$
\text { 75-03 }
$$

radical processes

$$
\text { 70-05 73-02 74-03 78-03 }
$$

radicals

68-01 70-05 71-03 72-01 72-02 73-02

74-01 74-03 74-04 75-02 77-01 78-01

78-03 79-01 79-02

(see hydrogen atom, hydroxyl radical,

solvated electrons, superoxide ion, specific

radical class i.e. alkyl radicals, biradicals,

halogen atoms, etc...)

rare-earth metal compunds

75-08

rare-earth metal ions

$76-01$

rhenium compounds

$$
\text { 74-02 }
$$

rhodium compounds

68-04: 75-08

ruthenium compounds

$$
\text { 74-02 75-08 78-05 }
$$

semiquinones

$$
\text { 75-03 78-03 }
$$

silanes

$$
\text { 74-04 }
$$

silver compounds

$$
\text { 75-08 }
$$

singlet oxygen

$$
\text { 75-04 76-02 76-04 76-05 79-03 79-04 }
$$

singlet states

64-01 67-04 69-02 69-03 69-04 70-01

70-02 70-04 71-02 73-05 73-06 73-08

73-09 73-10 75-05 75-06 75-07 76-03

77-02 77-04 77-06 78-02 78-04 78-06

(see singlet oxygen)

singlet-singlet transitions

67-04. 73-06

singlet-triplet transitions

$$
\text { 67-04. 73-06 }
$$

solid

$$
\begin{aligned}
& \text { 67-03 67-04 68-01 68-02 68-03 69-02 } \\
& \text { 69-03 69-04 70-01 70-03 71-02 72-01 } \\
& \text { 73-05 73-07 73-09 74-02 75-04 75-06 } \\
& \text { 75-07 75-08 77-03 77-04 77-05 }
\end{aligned}
$$

solvated electrons

$$
\text { 68-01 72-01 }
$$

(see hydrated electron)

superoxide ion

$$
\text { 77-01 }
$$

tantalum compounds

$$
\text { 74-02 }
$$


transition metal complexes

67-01 67-02 67-04 68-02 68-04 69-03 69-04 71-03 73-01 73-03 74-02 75-04

75-08 76-02 76-04 77-05 78-01 78-05 79-03

(see metal carbonyls, specific metal complex class i.e. cobalt compounds, iron compounds, manganese compounds. etc.....)

triplet states

64-01 67-04 68-03 69-01 69-02 69-03 69-04 70-01 70-02 70-04 71-01 71-02

72-02 73-04 73-05 73-06 73-08 73-09 73-10 75-04 75-05 75-06 75-07 76-01 76-03 77-02 77-04 77-05 77-06 78-02 78-04 78-06 triplet-triplet transitions

67-04 73-06 73-07 75-06

tungsten compounds

68-04 74-02

uranium compounds

78-05

vanadium compounds

74-02 75-08

zinc compounds

75-08

\section{Author Index}

Adams, G.E. - 73-02

Adamson, A.W. - 67-01 68-04

Anbar, M. - 67-05 73-01 75-02

Arnold, D.R. - 68-03

Azumi, T. - 69-03

Balzani, V. - 67-02 75-08 78-05

Bambenek, M. - 73-01

Becker, R.S. - 69-04

Bellus, D. - 76-04 79-03

Bensasson, R. - 78-06

Berces, T. - 72-03

Berlman, I.B. - 65-01 71-04 73-11

Birks, J.B. - 67-03 70-01 70-03 73-06 75-07

Bock, C.R. - 77-05

Boens, N. - 77-06

Bolletta, F. - 75-08 78-05

Buxton, G.V. - 78-01

Carassiti, V. - 67-02

Chapman, O.L. - 73-09

Cundall, R.B. - 75-05 77-04

Dalton, J.C. $-70-02$

De Mayo, P. - 71-01

De Schryver, F.C. - 77-06

Demyashkevich, A.B. - 77-02

Denisov, E.T. - 70-05 71-03 74-03

Dilling, W.L. - 69-01

Dorfman, L.M. - 73-02

Eigenmann, H.K. - 74-04

Encina, M.V. - 78-04

Engel, P.S. - 71-02 73-08

Ermolaev, V.L. - 75-04 76-01
Farhataziz - 75-02 77-01

Fleischauer, P.D. - 68-04

Fox, M.A. - 79-02

Gandolfi, M.T. - 78-05

Gollnick, K. - 76-05 79-04.

Habersbergerova, A. - 68-01 72-01

Hayon, E. - 74-01

Heinzelmann, W. - 73-07

Hendry, D.G. - 74-04

Howard, J.A. - 72-02 74-04

Ireland, J.F. - 76-03

Ivanov, V.B. - 76-02

Janovsky, 1. - 68-01 72-01

Khudyakov, I.V. - 75-03

Kinoshita, M. - 69-03

Kirk, A.D. - 73-03

Kirmse, W. - 73-10

Koerner von Gustorf, E.A. - 77-05

Kourim, P. - 72-01

Kuhn, H.J. - 79-04

Kuz'min, M.G. - 77-02

Kuz'min, V.A. - 75-03

Labhart, H. - 73-07

Land, E.J. - 78-06

Laurence, G.S. - 75-08

Legler, R. - 67-04

Lindholm, R.D. - 68-04

Lissi, E.A. - 78-04

Maciejewski, A. - 78-02

Maestri, M. - 78-05

Manfrin, M.F. - 75-08 
Martynov, I.Yu. - 77-02

Matuszewski, B. - 78-02

MeGlynn, S.P. - 69-03

Mill, T. - 74-04

Moggi, L. - 67-02 75-08

Monroe, B.M. - 71-02

Munro, I.H. - 67-03

Murov, S.L. - 73-05

Neta, P. - 67-05 79-01

Ogilvie, S.McD. - 75-05

Pereira, L.C. - 77-04.

Piszkiewicz, L. - 74-04

Put, J. - 77-06

Robinson, D.A. - 77-04

Ross, A.B. - 73-01 75-01 75-02 77-01 79-01

Scaiano, J.C. - 73-04 78-04

Scandola, F. - 67-02

Schmillen, A. - 67-04.

Schuchmann, H.-P. - 77-03

Sellers, R.M. - 78-01
Shakhverdov, T.A. - 75-04 76-01

Shlyapintokh, V.Ya. - 76-02

Simic, M. - 74-01

Steel, C. $-73-08$

Sveshnikova, E. - 75-04 76-01

Swallow, A.J. - 78-03

Teply, J. - 68-01

Turro, N.J. - 69-02 70-02

Uzhinov, B.M. - 77-02

Valentine, D.,Jr. - 68-02

Vander Donckt, E. - 70-04

von Sonntag, C. - 77-03

Waltz, W.L. - 68-04

Watts, D.W. - 68-04

Weiss, D.S. - 73-09

Wilkinson, F. - 64-01 75-06

Wrighton, M. - 74-02

Wyatt, P.A.H. - 76-03

Zinato, E. - 68-04

\section{Appendix: Review Series in Photochemistry}

Advances in Photochemistry, Editors: W.A. Noyes, Jr., G.S. Hammond and J.N. Pitts, Jr., John Wiley, Vol. 1(1963-) (11 Vol. Publ.).

Chemical and Biochemical Applications of Lasers, Editor: C. Bradley Moore, Academic Press, Vol. 1(1974-).

Creation and Detection of the Excited State, Editor: A.A. Lamola, Marcel Dekker, Vol. 1- (1971-).

Excited States, Editor: E.C. Lim, Academic Press, Vol. 1-(1974-).

Organic Photochemistry, Editor: A. Padwa, Marcel : Dekker, Vol. 1- (1967-) (9 Vol. Publ.).
Photochemical and Photobiological Reviews, Editor: K.C. Smith, Plenum, Vol. 1- (1976-) (4. Vol. Publ.).

Annual Survey of Photochemistry, Editors: N.J. Turro, and others, Wiley, Vol. 1-3 (1967-71).

Specialist Periodical Reports: Photochemistry, Senior Reporter, D. Bryce-Smith, London, The Chemical Society, Vol. 1- (1970-) (10 Vol. Publ.)

Highlights in the Literature, in: Molecular Photochemistry, Editors: J.C. Dalton, S.S. Hixon and D.C. Neckers, Marcel Dekker, Vol. 1- (1969-).

Yearly Review, in: Photochemistry and Photobiology, Editor: P.-S. Song, Pergamon Press, Vol. 1- (1963-).

2. U.S. GOVERNMENT PRINTING OFFICE : 1981 o-340-997 
TLE AND SUBTITLE NBS SP 578

Datalog of Data Compilations on Photochemical and photophysical Processes in Solution

5. Publication Date

Novem ber 1980

6. Performing Organization Code

8. Performing Organ. Report No.

\section{TTHOR(S)}

nes G. Brummer, W. Phillip Helman and Alberta B. Ross ERFORMING ORGANIZATION NAME AND ADDRESS adiation Chemistry Data Center adiation Laboratory niversity of Notre Dame otre Dame, IN 46556 PONSORING ORGANIZATION NAME AND COMPLETE ADDRESS (Street, City, State, ZIP) lational Bureau of Standards and Department of Energy vepartment of Commerce Washington, DC 20545 Jashington, DC 20234

Document describes a computer program; SF-185, FIPS Software Summary, is attached.

BSTRACT (A 200-word or less factual summary of most significant information. If document includes a significant bibliography or terature survey, mention it here.)

erences to compilations and reviews of data on photochemical and photophysical cesses in solution have been annotated to indicate subject and data content. exes are included for data types, keywords, and authors.

EY WORDS (six to twelve entries; alphabetical order; capitalize only the first letter of the first key word unless a proper name; sparated by semicolons)

a compilations; photochemistry; photophysics; review; solutions.

JAILABILITY

$$
\text { [x. Unlimited }
$$

For Official Distribution. Do Ilot Release to NTIS

¿ Order From Sup. of Doc., U.S. Government Printing Office, Washington, DC 20402

Order From National Technical Information Service (NTIS), Springfield, VA. 22161

\begin{tabular}{|l|c|}
\hline $\begin{array}{l}\text { 19. SECURITY CLASS } \\
\text { (THIS REPORT) }\end{array}$ & $\begin{array}{c}\text { 21. NO. OF } \\
\text { PRINTED PAGES } \\
27\end{array}$ \\
$\begin{array}{l}\text { UNCLASSIFIED } \\
\text { (THIS PAGE) }\end{array}$ & 22. Price \\
UNCLASSIFIED & $\$ 1.75$ \\
\hline
\end{tabular}


Where can you find all the reference data you need?

\section{Right in the} Journal of Physical and Chemical Reference Data!

Now in its sixth year, this valuable publication has proved that it fills the important gaps for you in the literature of the physical sciences.

Published by the American Institute of Physics and the American Chemical Society for the National Bureau of Standards, this quarterly gives you quantitative numerical data, with recommended values and uncertainty limits chosen by experts in the field.

Critical commentary on methods of measurement and sources of error, as well as full references to the original literature, is an integral part of each of your four issues a year.

Can you afford to be without this prime source of reliable data on physical and chemical properties? To start receiving your copies, just fill in the order form and drop into the mall. If you do use a purchase order, please attach the printed form as this will help us to expedite your order. Send for complete list of reprints!

\section{ta}

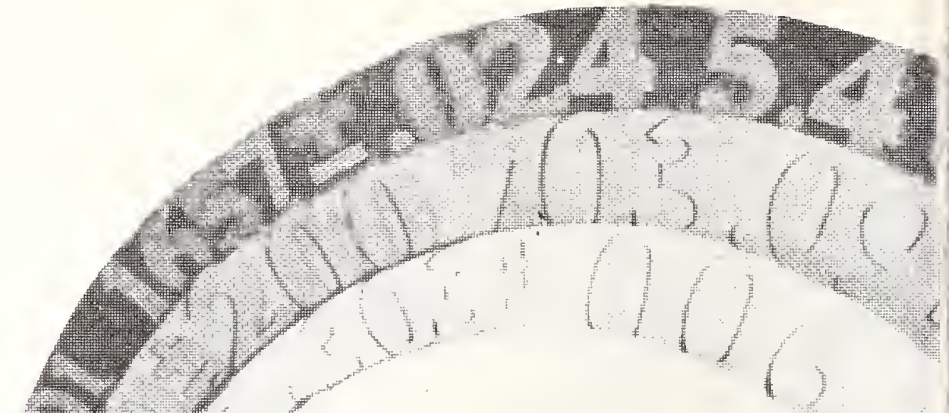

(1)

Journal of Physical and Chemical Reference Data American Chemical Society

1155 Sixteenth Street,N.W., Washington, D.C. 20036

Yes, I would like to receive the JOURNAL OF PHYSICAL AND CHEMICAL REFERENCE DATA at the one-year rate checked below

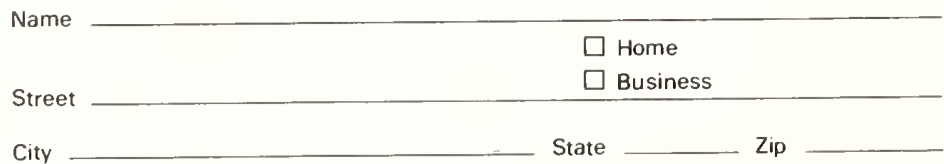

Bill me $\square \quad$ Bill company or school $\square \quad$ Payment enclosed $\square$

\section{Members Nonmembers \\ U.S. Canada, $\square \$ 25.00 \square \$ 100.00$ Mexico \\ Other Countries $\square \$ 29.00 \square \$ 104.00$}

Please Attach This Order Form To Purchase Order 


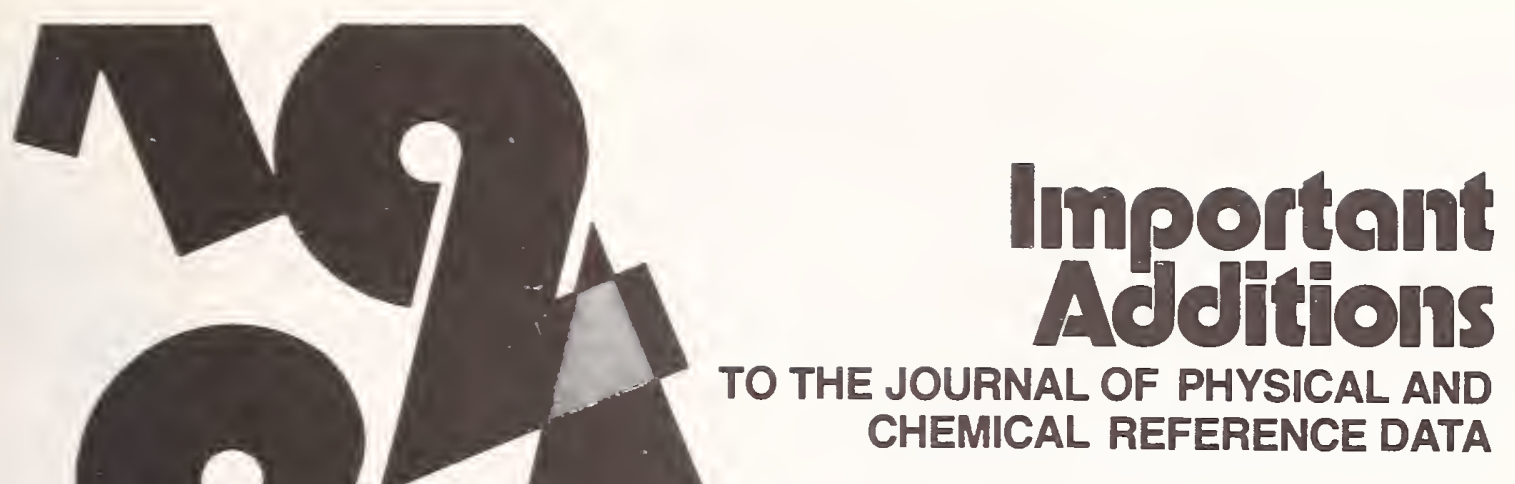

Three comprehensive reference volumes, each, as the Journal itself, published by the American Institute of Physics and the American Chemical Society for the National Bureau of Standards... your triple assurance of their accuracy, immediacy, and usefulness.

Supplement No. 1 to Vol. 2

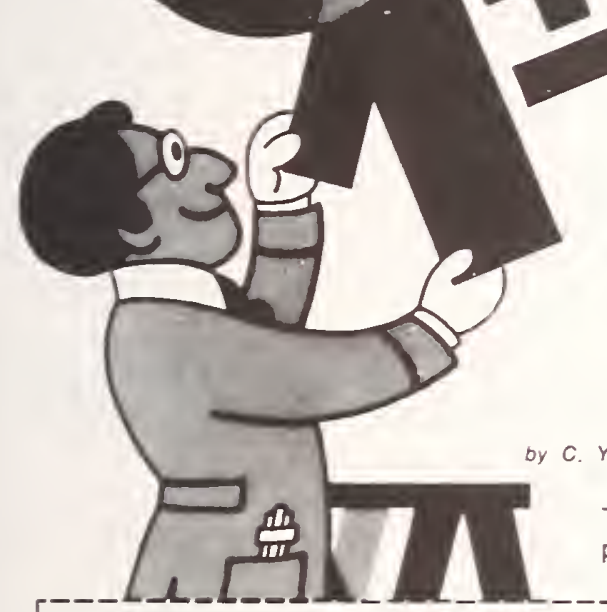
PROPERTIES OF ALIPHATIC ALCOHOLS"
and B. J. Zwofinski, Thermodynamics Research Center, Department of Chemistry. Texas A \& M Universily selected physical and thermodynamic properties of aliphatic alcohols that has been published in the world literature of chemistry.

Business Operations-Books and Journals Dept. American Chemical Society

115516 th Street, N.W.

Washington, O.C. 20036

Please send copies of at $\$$

A. "PHYSICAL AND THERMODYNAMIC PROPERTIES OF ALIPHATIC ALCOHOLS." (First supplement to Vol. 2 of the Journal of Physical and Chemical Relerence Data.) Hard Cover: $\$ 33.00$. Sott Cover: $\$ 30.00$.

8. "THERMAL CONDUCTIVITY OF THE ELEMENTS. A COMPREHENSIVE REVIEW." (First supplement to Vor. 3 of the Journal of Physical and Chemical Relerence Data.) Hard Cover $\$ 60.00$. Solt Cover: $\$ 55.00$

C. "ENERGETICS OF GASEOUS IONS." (First supplement to Vol. 6 of the Journal ot Physical and Chemical Reterence Data.J Hard Cover: $\$ 70.00$ Soll Cover: $\$ 65.00$.

$\square$ ' am enclosing a check $\square$ I am enclosing a money order

Name

Address

City ___ State___ Zip Code

Title Employer

Please add \$1.50 extra for forcign wostaye and handling.
Supplement No. 1 to Vol. 3

"THERMAL CONDUCTIVITY OF THE ELEMENTS:

A COMPREHENSIVE REVIEW"'

mophysicat Properties Research Center, Purdue University, West Lafayette, Indiana

This comprehensive review of the world's thermal conductivity data presents recommended or estimated values for all 105 elements.

Supplement No. 1 to Vot. 6 ENERGETICS OF GASEOUS IONS

by H. M. Rosenstock, K. Draxt, B. Steiner, and J. T. Herron, Nationaf Bureau of Standards

Provides a comprehensive body of critically evaluated information on ionization potentials, appearance potentials, electron affinities and heats of formation of gaseous positive and negative ions. It is a complete revision and extension of the earlier reference work, "Ionization Potentials, Appearance Potentials and Heats for Formation of Gaseous Positive Ions," NSRDS-NBS 26. 


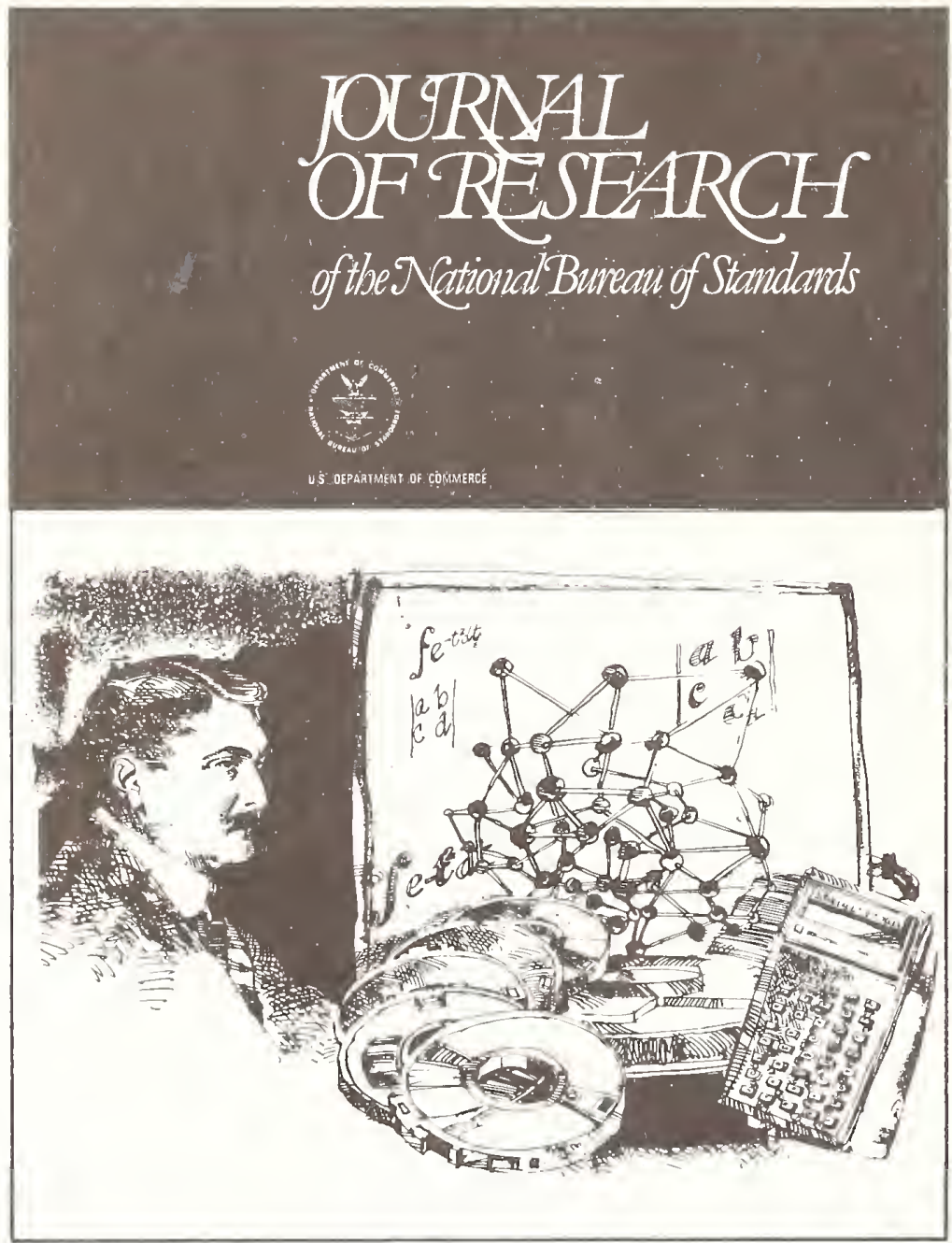

Subseribe nowThe new National Bureav of Standards Journal

The expanded Journal of Research of the Nationa Bureau of Standards reports NBS research and development in those disciplines of the physical and engineering sciences in which the Bureau is active. These include physics, chemistry, engine ing, mathematics, and computer sciences. Paper cover a broad range of subjects, with major emphasis on measurement methodology, and the basic technology underlying standardization. Als included from time to time are survey articles on topics closely related to the Bureau's technical and scientific programs. As a special service to subscribers each issue contains complete citatiol to all recent NBS publications in NBS and nonNBS media. Issued six times a year. Annual sub. scriptions: domestic $\$ 17.00$; foreign $\$ 21.25$. Sing copy, $\$ 3.00$ domestic; $\$ 3.75$ foreign.

- Note: The Journal was formerly published in two sections: Section A "Physics and Chemistry" and Section B "Mathematical Sciences."

NBS Board of Editors

Churchill Eisenhart,

Executive Editor (Mathematics)

John W. Cooper (Physics)

Donald D. Wagman (Chemistry)

Andrew J. Fowell (Engineering)

Joseph O. Harrison (Computer Science)

Howard J. M. Hanley (Boulder Labs.)

Subscription Order Form

Enter my subscription to NBS Journal of Research

at $\$ 17.00$. Add $\$ 4.25$ for foreign mailing. No additional postage is required for mailing within the United States or its possessions. (SJRA-File Code 2N)

Name-First, Last

Company Name or Additional Address Line

Street Address

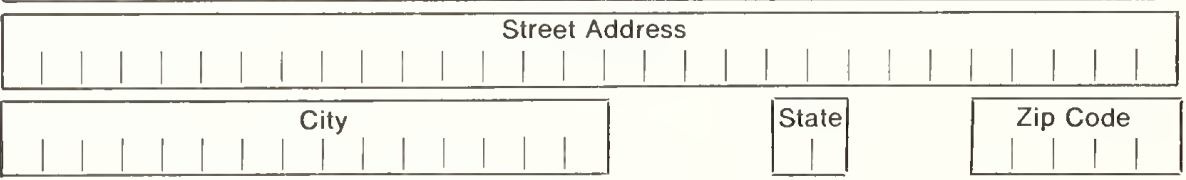

(or) COUNTRY

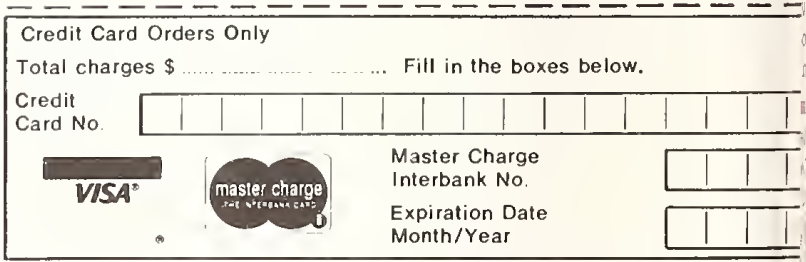

Remittance Enclosed. Domestic: Check or money order. Foreign: International money order, draft on an American or Canadian Bank, or by UNESCO coupons, Made payable to the Superintendent of Documents.

$\square$ Charge to my Deposit Account No.
MAIL ORDER FORM TO: Superintendent of Documents Government Printing Office Washington, D.C. 20402 


\section{PERIODICALS}

JOURNAL OF RESEARCH-The Journal of Research of the National Bureau of Standards reports NBS research and development in those disciplines of the physical and engineering sciences in which the Bureau is active. These include physics, chemistry, engineering, mathematics, and computer sciences. Papers cover a broad range of subjects, with major emphasis on measurement methodology and the basic technology underlying standardization. Also included from time to time are survey articles on topics closely related to the Bureau's technical and scientific programs. As a special service to subscribers each issue contains complete citations to all recent Bureau publications in both NBS and nonNBS media. Issued six times a year. Annual subscription: domestic \$13: foreign \$16.25. Single copy, \$3 domestic; $\$ 3.75$ foreign.

NOTE: The Journal was formerly published in two sections: Section A "Physics and Chemistry" and Section B "Mathematical Sciences."

DIMENSIONS/NBS-This monthly magazine is published to inform scientists, engineers, business and industry leaders, teachers, students, and consumers of the latest advances in science and technology, with primary emphasis on work at NBS. The magazine highlights and reviews such issues as energy research, fire protection, building technology, metric conversion, pollution abatement, health and safety, and consumer product performance. In addition, it reports the results of Bureau programs in measurement standards and techniques, properties of matter and materials, engineering standards and services, instrumentation, and automatic data processing. Annual subscription: domestic \$11; foreign $\$ 13.75$.

\section{NONPERIODICALS}

Monographs-Major contributions to the technical literature on various subjects related to the Bureau's scientific and technical activities.

Handbooks-Recommended codes of engineering and industrial practice (including safety codes) developed in cosperation with interested industries, professional organizations, and regulatory bodies.

Special Publications-Include proceedings of conferences spon. sored by NBS, NBS annual reports, and other special publications appropriate to this grouping such as wall charts, pocket cards, and bibliographies.

Applied Mathematics Series-Mathematical tables, manuals, and studies of special interest to physicists, engineers, chemists, biologists, mathematicians, computer programmers, and others engaged in scientific and technical work.

National Standard Reference Data Series-Provides quantitative data on the physical and chemical properties of materials, compiled from the world's literature and critically evaluated. Developed under a worldwide program coordinated by NBS under the authority of the National Standard Data Act (Public Law 90-396).
NOTE: The principal publication outlet for the foregoing data is the Journal of Physical and Chemical Reference Data (JPCRD) published quarterly for NBS by the American Chemical Society (ACS) and the American Institute of Physics (AIP). Subscriptions, reprints, and supplements available from ACS, 1155 Sixteenth St., NW, Washington, DC 20056.

Building Science Series-Disseminates technical information developed at the Bureau on building materials, components, systems, and whole structures. The series presents research results, test methods, and performance criteria related to the structural and environmental functions and the durability and safety characteristics of building elements and systems.

Technical Notes-Studies or reports which are complete in themselves but restrictive in their treatment of a subject. Analogous to monographs but not so comprehensive in scope or definitive in treatment of the subject area. Often serve as a vehicle for final reports of work performed at NBS under the sponsorship of other government agencies.

Voluntary Product Standards-Developed under procedures published by the Department of Commerce in Part 10, Title 15, of the Code of Federal Regulations. The standards establish nationally recognized requirements for products, and provide all concerned interests with a basis for common understanding of the characteristics of the products. NBS administers this program as a supplement to the activities of the private sector standardizing organizations.

Consumer Information Series-Practical information, based on NBS research and experience, covering areas of interest to the consumer. Easily understandable language and illustrations provide useful background knowledge for shopping in today's technological marketplace.

Order the above NBS publications from: Superintendent of Documents, Government Printing Office, Washington, DC 20402.

Order the following NBS publications-FIPS and NBSIR's-from the National Technical Information Services, Springfield, VA 22161.

Federal Information Processing Standards Publications (FIPS PUB)-Publications in this series collectively constitute the Federal Information Processing Standards Register. The Register serves as the official source of information in the Federal Government regarding standards issued by NBS pursuant to the Federal Property and Administrative Services Act of 1949 as amended, Public Law 89-306 (79 Stat. 1127), and as implemented by Executive Order 11717 (38 FR 12315, dated May 11, 1973) and Part 6 of Title 15 CFR (Code of Federal Regulations).

NBS Interagency Reports (NBSIR) - A special series of interim or final reports on work performed by NBS for outside sponsors (both government and non-government). In general, initial distribution is handled by the sponsor; public distribution is by the National Technical Information Services, Springfield, VA 22161, in paper copy or microfiche form.

\section{BIBLIOGRAPHIC SUBSCRIPTION SERVICES}

The following current-awareness and literature-survey bibliographies are issued periodically by the Bureau:

Cryogenic Data Center Current A wareness Service. A literature survey issued biweekly. Annual subscription: domestic \$35; foreign $\$ 45$.

Liquefied Natural Gas. A literature survey issued quarterly. Annual subscription: $\$ 30$.
Superconducting Devices and Materials. A literature survey issued quarterly. Annual subscription: \$45. Please send subscription orders and remittances for the preceding bibliographic services to the National Bureau of Standards, Cryogenic Data Center (736) Boulder, CO 80303. 
U.S. DEPARTMENT OF COMMERCE

National Bureau of Standards

Washington, D.C. 20234

PDSTAGE ANO FEES PAID U.S. DEPAATMENT OF CDMMEACE

COM-2I5

OFFICIAL BUSINESS

Penalty for Private Use, $\$ 300$ 